\title{
The inner solar corona seen by SUMER, LASCO/C1, and EIT: Electron densities and temperatures during the rise of the new solar cycle
}

\author{
K. Wilhelm ${ }^{1}$, B. Inhester ${ }^{1}$, and J. S. Newmark ${ }^{2}$ \\ 1 Max-Planck-Institut für Aeronomie, Max-Planck-Straße 2, 37191 Katlenburg-Lindau, Germany \\ 2 Naval Research Laboratories, 4555 Overlook Ave., SW, Washington, DC 20375, USA
}

Received 7 August 2001 / Accepted 9 November 2001

\begin{abstract}
Detailed investigations of the corona and the source regions of the solar wind have become possible with spectroscopic and imaging instruments on the Solar and Heliospheric Observatory (SOHO). We present observations in the extreme ultraviolet (EUV), pertinent to the generation of the slow solar wind, which were obtained by the Solar Ultraviolet Measurements of Emitted Radiation (SUMER) spectrograph and by the Extreme-ultraviolet Imaging Telescope (EIT) in early 1998 under relatively quiet solar conditions, but with several active regions of the new solar cycle present. At the same time, forbidden iron lines in the visible were observed by the Large-Angle Spectroscopic Coronagraph (LASCO/C1). We study, in particular, the plasma parameters and the spatial structures of the low-altitude streamer regions, and find an electron density of $n_{\mathrm{e}} \approx 2 \times 10^{8} \mathrm{~cm}^{-3}$ at $5 \mathrm{Mm}$ above the equatorial limb for the coronal plasma, and $n_{\mathrm{e}}=(2$ to 6$) \times 10^{9} \mathrm{~cm}^{-3}$ for the plasma at transition-region temperatures. High-temperature regions have been found at mid-latitudes with electron temperatures of $T_{\mathrm{e}} \approx 1.4 \times 10^{6} \mathrm{~K}$ at heights of about $80 \mathrm{Mm}$ and lower temperatures near the equator.
\end{abstract}

Key words. Sun: atmosphere - Sun: transition region - Sun: corona - Sun: UV radiation

\section{Introduction}

The solar corona at low and medium latitudes is occupied by closed magnetic field structures during the minimum of the solar sunspot cycle and yet has been identified as being the source region of the slow solar wind (e.g., Schwenn et al. 1997; Raymond et al. 1997). In addition, many coronal mass ejections originate in this latitudinal range even when the magnetic activity is low (e.g., Dere et al. 1997; Simnett et al. 1997; Plunkett et al. 1997; Wu et al. 1997; Vourlidas et al. 2000). In contrast, coronal holes have been shown to accelerate the fast solar wind (Krieger et al. 1973; Schwenn 1990; Geiss et al. 1995; Woch et al. 1997; Hassler et al. 1999; Cranmer et al. 1999; Wilhelm et al. 2000a). During the last activity minimum between the sunspot cycles 22 and 23, well-developed quiescent equatorial streamers and polar coronal holes provided excellent opportunities for SOHO - the Solar and Heliospheric Observatory of ESA and NASA - to study these phenomena in their undisturbed or mildly disturbed configurations. In this contribution, we will focus our attention on the plasma parameters electron density and temperature in the closed

Send offprint requests to: K. Wilhelm, e-mail: wilhelm@linmpi.mpg.de magnetic field region at low and medium solar latitudes in early 1998 during the early rise of the new solar cycle. The SUMER, LASCO/C1, and EIT instruments on SOHO obtained the data to be presented in the following sections. The instruments have been described elsewhere (Brueckner et al. 1995; Delaboudinière et al. 1995 Wilhelm et al. 1995, 1997a; Lemaire et al. 1997; Moses et al. 1997; Schwenn et al. 1997), and the reader is referred to these publications for instrumental details.

\section{Observations and data analysis}

\subsection{SUMER}

The SUMER spectrometer observed the low corona $(r<$ $1.6 R_{\odot}$, where $r$ is the radius vector of the observation site in the plane of the sky and $1 R_{\odot}=696000 \mathrm{~km}$ is the solar radius) on many occasions, and results on the findings relevant in the context of the present discussion have been published, e.g., by Feldman et al. (1997, 2000), Doschek et al. (1997), Feldman (1998), and Wilhelm et al. (1998, 2000). Here we present spectra obtained on complete cross sections of the east and west equatorial streamers near the Sun in February 1998 in an attempt to determine the plasma conditions in this part of the corona. This is the 


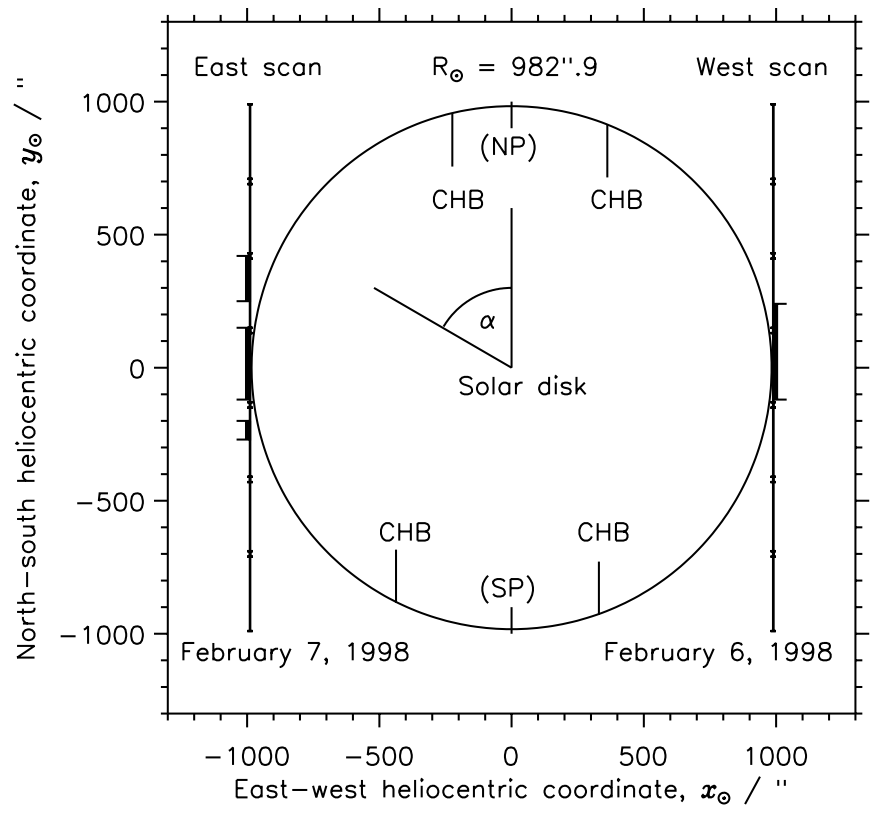

Fig. 1. Geometry of the SUMER observations on north-south cross sections of the solar east and west streamers obtained on February $6 / 7,1998$. The positions of the individual slit settings are indicated along the cross sections. Also shown by heavy bars are the regions with radiation from transition region or prominence lines. The angular extent of the solar radius at the time of observation is given and the approximate locations of the coronal hole boundaries (CHB) near the limb are marked from EIT images. Also defined is the position angle, $\alpha$. For details of the coronal structure during the SUMER observations see Figs. 5, 9, 10 and 12 .

only set available of such spectra. The geometry of the observing scheme is shown in Fig. 1, and more information on the observational programmes is given in Table 1. Based on data obtained during the same period, a study of the neon-to-magnesium elemental abundance variations has already been published (Wilhelm 1999).

Not all of the spectra observed in the wavelength range from $702 \AA$ to $1468 \AA$ in first order of diffraction with superimposed second order spectra could be analysed here, but we studied those wavelength intervals in detail, which could shed light on the important plasma parameters electron density and electron temperature. Consequently, we looked at the ratio of the Si VIII $(\lambda \lambda 1440,1445)$ forbidden lines for electron density diagnostics (Doschek et al. $1997)$, at the ratio of the Mg IX $(\lambda \lambda 706,750)$ lines for electron temperature diagnostics (Keenan et al. 1984; Doyle et al. 1985; Wilhelm et al. 1998). Of particular interest was the wavelength range from $758 \AA$ to $793 \AA$ containing the Ne VIII resonant lines at $770 \AA$ and $780 \AA$ and several $\mathrm{Mg}$ VIII lines, in addition, to many transition region lines (N II, N III, N IV, S v, O IV, and O v) with temperatures of the peak ionic fractions between $2.9 \times 10^{4} \mathrm{~K}$ and $2.4 \times 10^{5} \mathrm{~K}$ and some lines of the Sx and S XI spectra. The emission lines treated here and their characteristics are compiled in Table 2. The wavelengths observed with SUMER, $\lambda_{\text {obs }}$, are not the most accurate determinations possible, but are
Table 1. Dates and pointing positions of the relevant SUMER observations. The sampling time at each position was $300 \mathrm{~s}$ with a duration ${ }^{a}$ of $1 \mathrm{~h} 13 \mathrm{~min}$ for a spectral $\operatorname{scan}^{b}$ through 12 selected bands on both photocathodes ${ }^{c}$. A slit with angular dimensions of $1^{\prime \prime} \times 300^{\prime \prime}$ was used $\left(1^{\prime \prime} \stackrel{\wedge}{=} 708 \mathrm{~km}\right.$ on the Sun).

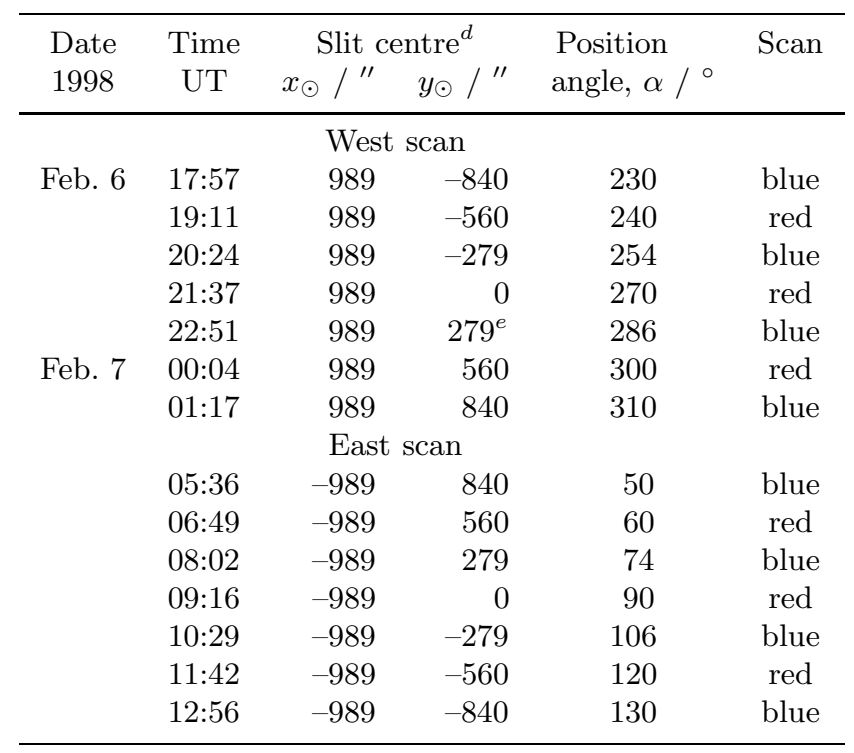

${ }^{a}$ With overhead for spectrometer settings.

${ }^{b}$ The direction of the wavelength scan is given in the last column: blue towards shorter wavelengths; red towards longer wavelengths.

${ }^{c}$ Photocathodes: Bare microchannel plate, potassium bromide (KBr).

${ }^{d}$ Nominal pointing positions.

${ }^{e} \mathrm{Mg}$ IX lines not available (telemetry gap).

only evaluated to allow a reliable line identification. The corresponding normalized contribution functions, $G_{\mathrm{n}}\left(T_{\mathrm{e}}\right)$ (cf. Mariska 1992; Feldman et al. 2000), valid for ionization equilibrium, are shown in Fig. 2 using interpolated data of Arnaud \& Rothenflug (1985) as well as Arnaud \& Raymond (1992). Note the high-temperature tails of the lines HeII, O vi, Ar VIII, and Ne VIII from hydrogen-like and lithium-like ions.

The full detector format of 360 (spatial) $\times 1024$ (spectral) pixels has been telemetered to the ground, geometrically corrected and radiometrically calibrated with the help of standard SUMER analysis software, before any binning in the spatial direction was performed for some of the presentations. Since substantial binning was required in the dark regions of the corona, a flat-field correction was not applied there nor were deadtime or gain-depression corrections needed because of the low count rates encountered (with a maximum of 5 count $\mathrm{s}^{-1}$ pixel $^{-1}$ ).

The data of different exposures have then been pasted together by eliminating small overlaps of $20^{\prime \prime}$ between individual slit images. This has not in all cases produced a perfect fit, presumably because of temporal variations within the exposure time of more than one hour for each slit position. Figure 3 shows the cross section of the west streamer 
Table 2. Emission lines observed in first order of diffraction with SUMER detector B in the wavelength range from $758 \AA$ to $793 \AA$ (as well as some other lines of interest) together with their temperatures of maximum ionic abundance, $T_{\mathrm{m}}$, and formation temperatures, $T_{\mathrm{C}}$. Literature wavelengths are given after Kelly (1987), unless a reference is explicitly stated. Ground levels are indicated by "g".

\begin{tabular}{|c|c|c|c|c|c|c|c|}
\hline \multicolumn{2}{|c|}{ Wavelength } & \multirow[t]{2}{*}{ Line } & \multicolumn{3}{|c|}{ Transition } & \multicolumn{2}{|c|}{ Temperatures } \\
\hline$\lambda_{\text {obs }}^{a, b} / \AA$ & $\lambda_{\text {lit }} / \AA$ & & & & & $T_{\mathrm{m}}^{c} / \mathrm{K}$ & $T_{\mathrm{C}} / \mathrm{K}$ \\
\hline 700.24 & 700.245 & Ar VIII & g $3 \mathrm{~s}^{2} \mathrm{~S}_{1 / 2}$ & - & $3 \mathrm{p}^{2} \mathrm{P}_{3 / 2}$ & $3.6 \times 10^{5}$ & $3.6 \times 10^{5}$ \\
\hline 706.06 & $706.06^{d}$ & Mg IX & g $2 \mathrm{~s}^{2}{ }^{1} \mathrm{~S}_{0}$ & - & $2 \mathrm{~s} 2 \mathrm{p}^{3} \mathrm{P}_{1}$ & $9.5 \times 10^{5}$ & $9.5 \times 10^{5}$ \\
\hline 749.55 & 749.55 & Mg IX & $2 \mathrm{~s} 2 \mathrm{p}{ }^{1} \mathrm{P}_{1}$ & - & $2 \mathrm{p}^{2}{ }^{1} \mathrm{D}_{2}$ & $9.5 \times 10^{5}$ & $9.5 \times 10^{5}$ \\
\hline 758.68 & 758.678 & $\mathrm{OV}$ & $2 \mathrm{~s} 2 \mathrm{p}^{3} \mathrm{P}_{1}$ & - & $2 \mathrm{p}^{2}{ }^{3} \mathrm{P}_{2}$ & $2.4 \times 10^{5}$ & $2.5 \times 10^{5}$ \\
\hline 759.44 & 759.441 & $\mathrm{OV}(\mathrm{S}$ IV $)$ & $2 \mathrm{~s} 2 \mathrm{p}{ }^{3} \mathrm{P}_{0}$ & - & $2 \mathrm{p}^{2}{ }^{3} \mathrm{P}_{1}$ & $2.4 \times 10^{5}$ & $2.5 \times 10^{5}$ \\
\hline 760.23 & 760.228 & $\mathrm{Ov}$ & $2 \mathrm{~s} 2 \mathrm{p}{ }^{3} \mathrm{P}_{1}$ & - & $2 \mathrm{p}^{2}{ }^{3} \mathrm{P}_{1}$ & $2.4 \times 10^{5}$ & $2.5 \times 10^{5}$ \\
\hline 760.45 & 760.445 & $\mathrm{Ov}$ & $2 \mathrm{~s} 2 \mathrm{p}^{3} \mathrm{P}_{2}$ & - & $2 \mathrm{p}^{2}{ }^{3} \mathrm{P}_{2}$ & $2.4 \times 10^{5}$ & $2.5 \times 10^{5}$ \\
\hline 761.12 & 761.128 & $\mathrm{OV}_{\mathrm{V}}$ & $2 \mathrm{~s} 2 \mathrm{p}{ }^{3} \mathrm{P}_{1}$ & - & $2 \mathrm{p}^{2}{ }^{3} \mathrm{P}_{0}$ & $2.4 \times 10^{5}$ & $2.5 \times 10^{5}$ \\
\hline 762.00 & 762.003 & $\mathrm{Ov}$ & $2 \mathrm{~s} 2 \mathrm{p}^{3} \mathrm{P}_{2}$ & - & $2 \mathrm{p}^{2}{ }^{3} \mathrm{P}_{1}$ & $2.4 \times 10^{5}$ & $2.5 \times 10^{5}$ \\
\hline 762.65 & $762.66^{e}$ & Mg VIII & g $2 s^{2} 2 p^{2} P_{1 / 2}$ & - & $2 \mathrm{~s} 2 \mathrm{p}^{2}{ }^{4} \mathrm{P}_{3 / 2}$ & $8.1 \times 10^{5}$ & $8.1 \times 10^{5}$ \\
\hline 763.34 & 763.340 & $\mathrm{~N}$ III & g $2 s^{2} 2 p^{2} P_{1 / 2}$ & - & $2 \mathrm{~s} 2 \mathrm{p}^{2}{ }^{2} \mathrm{~S}_{1 / 2}$ & $7.9 \times 10^{4}$ & $1.0 \times 10^{5}$ \\
\hline 764.37 & 764.357 & N III & $2 \mathrm{~s}^{2} 2 \mathrm{p}^{2} \mathrm{P}_{3 / 2}$ & - & $2 \mathrm{~s} 2 \mathrm{p}^{2}{ }^{2} \mathrm{~S}_{1 / 2}$ & $7.9 \times 10^{4}$ & $1.0 \times 10^{5}$ \\
\hline 765.15 & 765.148 & N IV & g $2 \mathrm{~s}^{2}{ }^{1} \mathrm{~S}_{0}$ & - & $2 \mathrm{~s} 2 \mathrm{p}{ }^{1} \mathrm{P}_{1}$ & $1.4 \times 10^{5}$ & $1.5 \times 10^{5}$ \\
\hline 769.38 & $769.36^{e}$ & Mg VIII & g $2 s^{2} 2 p^{2} P_{1 / 2}$ & - & $2 \mathrm{~s} 2 \mathrm{p}^{2}{ }^{4} \mathrm{P}_{1 / 2}$ & $8.1 \times 10^{5}$ & $8.1 \times 10^{5}$ \\
\hline 770.41 & $770.428^{f}$ & Ne VIII & g $2 \mathrm{~s}^{2} \mathrm{~S}_{1 / 2}$ & - & $2 \mathrm{p}^{2} \mathrm{P}_{3 / 2}$ & $6.2 \times 10^{5}$ & $6.2 \times 10^{5}$ \\
\hline 771.87 & 771.901 & N III & $2 \mathrm{~s} 2 \mathrm{p}^{2}{ }^{4} \mathrm{P}_{3 / 2}$ & - & $2 \mathrm{p}^{3}{ }^{4} \mathrm{~S}_{3 / 2}$ & $7.9 \times 10^{4}$ & $1.0 \times 10^{5}$ \\
\hline 772.28 & $772.26^{e}$ & Mg VIII & $2 \mathrm{~s}^{2} 2 \mathrm{p}^{2} \mathrm{P}_{3 / 2}$ & - & $2 \mathrm{~s} 2 \mathrm{p}^{2}{ }^{4} \mathrm{P}_{5 / 2}$ & $8.1 \times 10^{5}$ & $8.1 \times 10^{5}$ \\
\hline 772.54 & $772.7^{g}$ & Al VIII & $2 \mathrm{~s}^{2} 2 \mathrm{p}^{2}{ }^{3} \mathrm{P}_{2}$ & - & $2 \mathrm{~s} 2 \mathrm{p}^{3}{ }^{5} \mathrm{~S}_{2}$ & $7.9 \times 10^{5}$ & $7.9 \times 10^{5}$ \\
\hline 774.53 & 774.518 & $\mathrm{Ov}$ & $2 \mathrm{~s} 2 \mathrm{p}{ }^{1} \mathrm{P}_{1}$ & - & $2 \mathrm{p}^{2}{ }^{2} \mathrm{~S}_{0}$ & $2.4 \times 10^{5}$ & $2.5 \times 10^{5}$ \\
\hline 775.41 & - & $?$ & & & & & \\
\hline 775.99 & 775.965 & N II & $2 \mathrm{~s}^{2} 2 \mathrm{p}^{2}{ }^{1} \mathrm{D}_{2}$ & - & $2 \mathrm{~s} 2 \mathrm{p}^{3}{ }^{1} \mathrm{D}_{2}$ & $2.9 \times 10^{4}$ & $6.2 \times 10^{4}$ \\
\hline 776.24 & $776.37^{h}$ & $\mathrm{Sx}$ & g $2 s^{2} 2 p^{3}{ }^{4} S_{3 / 2}$ & - & $2 \mathrm{~s}^{2} 2 \mathrm{p}^{3}{ }^{2} \mathrm{P}_{3 / 2}$ & $1.3 \times 10^{6}$ & $1.3 \times 10^{6}$ \\
\hline 779.88 & 779.734 & O IV & $2 \mathrm{~s} 2 \mathrm{p}^{2}{ }^{2} \mathrm{D}_{5 / 2}$ & - & $2 \mathrm{p}^{3}{ }^{2} \mathrm{D}_{3 / 2}$ & $1.7 \times 10^{5}$ & $1.8 \times 10^{5}$ \\
\hline bl & 779.821 & O IV & $2 \mathrm{~s} 2 \mathrm{p}^{2}{ }^{2} \mathrm{D}_{3 / 2}$ & - & $2 \mathrm{p}^{3}{ }^{2} \mathrm{D}_{3 / 2}$ & $1.7 \times 10^{5}$ & $1.8 \times 10^{5}$ \\
\hline $\mathrm{bl}$ & 779.912 & O IV & $2 \mathrm{~s} 2 \mathrm{p}^{2}{ }^{2} \mathrm{D}_{5 / 2}$ & - & $2 \mathrm{p}^{3}{ }^{2} \mathrm{D}_{5 / 2}$ & $1.7 \times 10^{5}$ & $1.8 \times 10^{5}$ \\
\hline bl & 779.997 & O IV & $2 \mathrm{~s} 2 \mathrm{p}^{2}{ }^{2} \mathrm{D}_{3 / 2}$ & - & $2 \mathrm{p}^{3}{ }^{2} \mathrm{D}_{5 / 2}$ & $1.7 \times 10^{5}$ & $1.8 \times 10^{5}$ \\
\hline 780.32 & 780.324 & Ne VIII & g $2 \mathrm{~s}^{2} \mathrm{~S}_{1 / 2}$ & - & $2 \mathrm{p}^{2} \mathrm{P}_{1 / 2}$ & $6.2 \times 10^{5}$ & $6.2 \times 10^{5}$ \\
\hline 782.36 & $782.36^{e}$ & Mg VIII & $2 \mathrm{~s}^{2} 2 \mathrm{p}^{2} \mathrm{P}_{3 / 2}$ & - & $2 \mathrm{~s} 2 \mathrm{p}^{2}{ }^{4} \mathrm{P}_{3 / 2}$ & $8.1 \times 10^{5}$ & $8.1 \times 10^{5}$ \\
\hline 782.91 & $782.96^{i}$ & S XI & $2 \mathrm{~s}^{2} 2 \mathrm{p}^{2}{ }^{3} \mathrm{P}_{1}$ & - & $2 \mathrm{~s}^{2} 2 \mathrm{p}^{2}{ }^{1} \mathrm{~S}_{0}$ & $1.7 \times 10^{6}$ & $1.8 \times 10^{6}$ \\
\hline 786.47 & $786.470^{j}$ & $\mathrm{~S} v$ & g $3 \mathrm{~s}^{2}{ }^{1} \mathrm{~S}_{0}$ & - & $3 \mathrm{~s} 3 \mathrm{p}^{1} \mathrm{P}_{1}$ & $1.5 \times 10^{5}$ & $1.5 \times 10^{5}$ \\
\hline 787.44 & $787.56^{h}$ & $\mathrm{Sx}$ & g $2 s^{2} 2 p^{3}{ }^{4} S_{3 / 2}$ & - & $2 \mathrm{~s}^{2} 2 \mathrm{p}^{3}{ }^{2} \mathrm{P}_{1 / 2}$ & $1.3 \times 10^{6}$ & $1.3 \times 10^{6}$ \\
\hline 787.74 & 787.711 & O IV & g $2 s^{2} 2 p^{2} P_{1 / 2}$ & - & $2 \mathrm{~s} 2 \mathrm{p}^{2}{ }^{2} \mathrm{D}_{3 / 2}$ & $1.7 \times 10^{5}$ & $1.8 \times 10^{5}$ \\
\hline 789.44 & $789.41^{e}$ & Mg VIII & $2 \mathrm{~s}^{2} 2 \mathrm{p}^{2} \mathrm{P}_{3 / 2}$ & - & $2 \mathrm{~s} 2 \mathrm{p}^{2}{ }^{4} \mathrm{P}_{1 / 2}$ & $8.1 \times 10^{5}$ & $8.1 \times 10^{5}$ \\
\hline 789.78 & $789.78^{e}$ & Na ViII & g $2 \mathrm{~s}^{2}{ }^{1} \mathrm{~S}_{0}$ & - & $2 \mathrm{~s} 2 \mathrm{p}^{3} \mathrm{P}_{1}$ & $7.1 \times 10^{5}$ & $7.1 \times 10^{5}$ \\
\hline 790.19 & 790.199 & O IV & $2 \mathrm{~s}^{2} 2 \mathrm{p}^{2} \mathrm{P}_{3 / 2}$ & - & $2 \mathrm{~s} 2 \mathrm{p}^{2}{ }^{2} \mathrm{D}_{5 / 2}$ & $1.7 \times 10^{5}$ & $1.8 \times 10^{5}$ \\
\hline 1031.93 & 1031.924 & O VI & g $2 \mathrm{~s}^{2} \mathrm{~S}_{1 / 2}$ & - & $2 \mathrm{p}^{2} \mathrm{P}_{3 / 2}$ & $3.0 \times 10^{5}$ & $3.0 \times 10^{5}$ \\
\hline 1037.60 & 1037.614 & O VI & g $2 \mathrm{~s}^{2} \mathrm{~S}_{1 / 2}$ & - & $2 \mathrm{p}^{2} \mathrm{P}_{1 / 2}$ & $3.0 \times 10^{5}$ & $3.0 \times 10^{5}$ \\
\hline 1440.49 & 1440.49 & Si VIII & g $2 s^{2} 2 p^{3}{ }^{4} S_{3 / 2}$ & - & $2 \mathrm{~s}^{2} \mathrm{p}^{3}{ }^{2} \mathrm{D}_{5 / 2}$ & $7.9 \times 10^{5}$ & $7.9 \times 10^{5}$ \\
\hline 1445.75 & 1445.76 & Si VIII & $\mathrm{g} 2 \mathrm{~s}^{2} 2 \mathrm{p}^{3}{ }^{4} \mathrm{~S}_{3 / 2}$ & - & $2 \mathrm{~s}^{2} \mathrm{p}^{3}{ }^{2} \mathrm{D}_{3 / 2}$ & $7.9 \times 10^{5}$ & $7.9 \times 10^{5}$ \\
\hline
\end{tabular}

${ }^{a}$ Feldman et al. (1997); ${ }^{b}$ Curdt et al. (1997); ${ }^{c}$ Arnaud \& Rothenflug (1985); ${ }^{d}$ Ridgeley \& Burton (1972); ${ }^{e}$ Edlén (1983);

${ }^{f}$ Dammasch et al. (1999); ${ }^{g}$ Denne \& Hinnov (1984); ${ }^{h}$ Edlén (1984); ${ }^{i}$ Edlén (1985); ${ }^{j}$ Kaufman \& Martin (1993).

near the Ne VIII lines as negative display. Here and in the other figures below we use a reversed grey scale code which shows emission intensity in dark and the absence of emission in white. In Fig. 4 we present the corresponding spectra obtained in the east streamer. A spatial binning of four pixels has been applied for these overviews. Both streamer spectra are very similar with the exception of emission regions at north-south heliocentric coordinates, $y_{\odot}=-220^{\prime \prime}$ to $-279^{\prime \prime}$ and $250^{\prime \prime}$ to $420^{\prime \prime}$, which are only present in the eastern hemisphere. The transition region lines in the northern range exhibit pronounced Doppler shifts towards long wavelengths (red shift) with respect to the undisturbed lines, which amount to line-of-sight (LOS) speeds of $\approx 37 \mathrm{~km} \mathrm{~s}^{-1}$ away from SOHO, if the lines outside this range are assumed to be at rest. This is a safe assumption as the light seen in these lines at the northern and southern slit positions is essential straylight from the solar disk, which cannot be red shifted by such an amount. By inspecting the He II image of EIT for this day shown in Fig. 5, a large prominence can be identified as cause of 


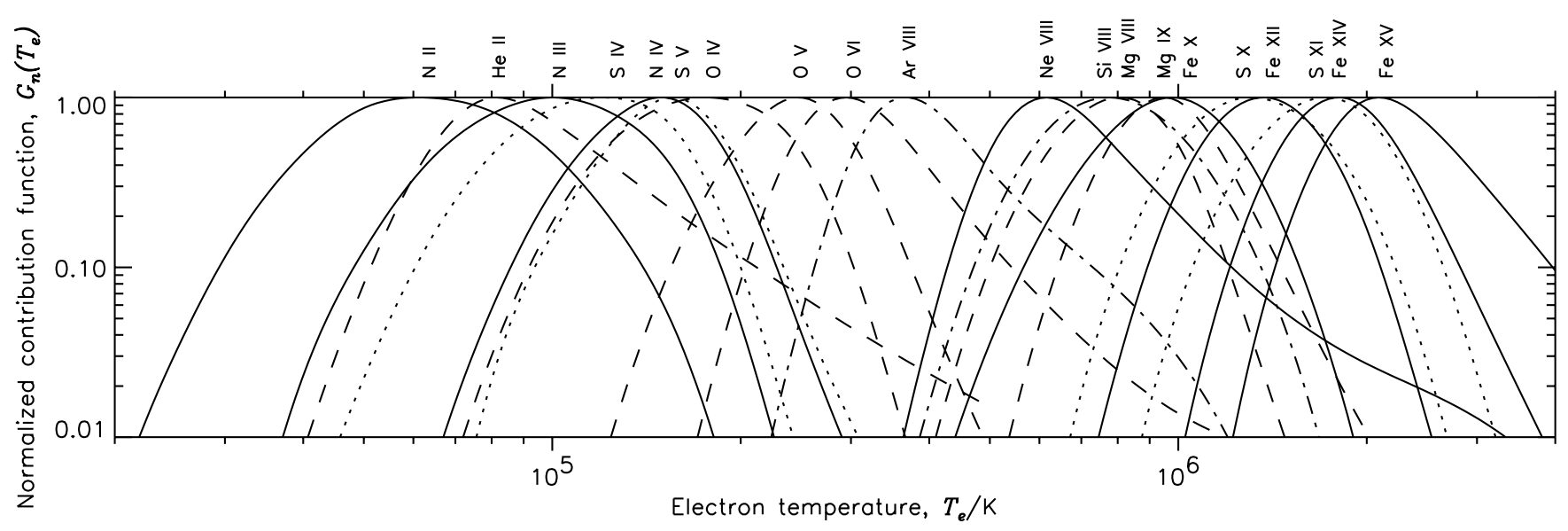

Fig. 2. Normalized contribution functions of the emission lines discussed in this paper. The ionic fractions except for the iron ions are taken from Arnaud \& Rothenflug (1985). The data for iron are from Arnaud \& Raymond (1992).

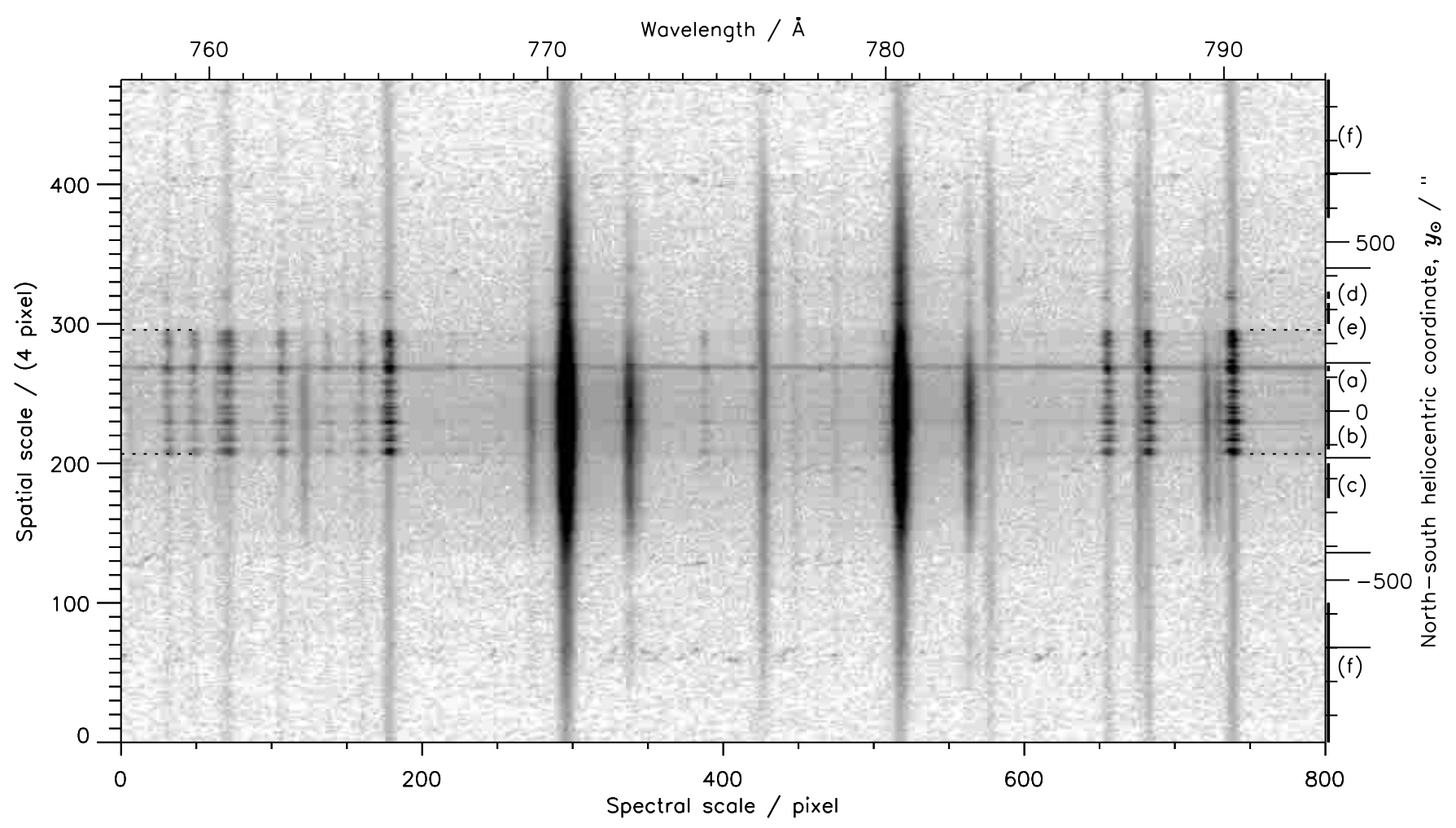

Fig. 3. North-south cross section of the west streamer in the wavelength range from $758 \AA$ to $793 \AA$ (part of Band 3 out of 12 ; cf. Table 1). The geometry can be seen from Fig. 1. The data have been binned in the spatial direction by a factor of 4 . On the right-hand side, the different slit positions are indicated by long tick marks. Highlighted by vertical bars and identified by lower case letters are spatial regions for which we show amplitude spectra in Fig. 7, where most of the lines in the spectral range shown are identified. Extremely sharp boundaries can be seen in all transition region lines and in the H I Lyman continuum at $y_{\odot}=-127^{\prime \prime}$ and $240^{\prime \prime}$, indicated by dotted marks. The streamer is relatively quiet and displays strong spatial brightness variations only in transition region lines in the central regions (a) and (b). Some cloudy patches visible at $y \odot=680^{\prime \prime}, 430^{\prime \prime}$, $-420^{\prime \prime}$, and $-720^{\prime \prime}$ are noise signals stemming from the detector in its high-gain setting. The central portion with full spatial resolution has been presented elsewhere by Wilhelm (1999).

the cool moving plasma north of the solar equator. Note that the coronal lines do not show any specific Doppler shifts in the prominence region, which can best be seen for the Sx ( $\lambda 787)$ line in relation to the O IV $(\lambda 787)$ line or the three lines near $790 \AA$ (Mg VIII, Na VIII, and O IV).
This prominence is also visible in the Ca II K3 long exposure of Meudon, whereas no such activity is present at the western slit positions. There is however a weak prominence visible in the He II image of EIT at $y_{\odot} \approx 120^{\prime \prime}$ to $250^{\prime \prime}$. This may cause the slight asymmetry in Fig. 3 with respect to the point of closest approach. 


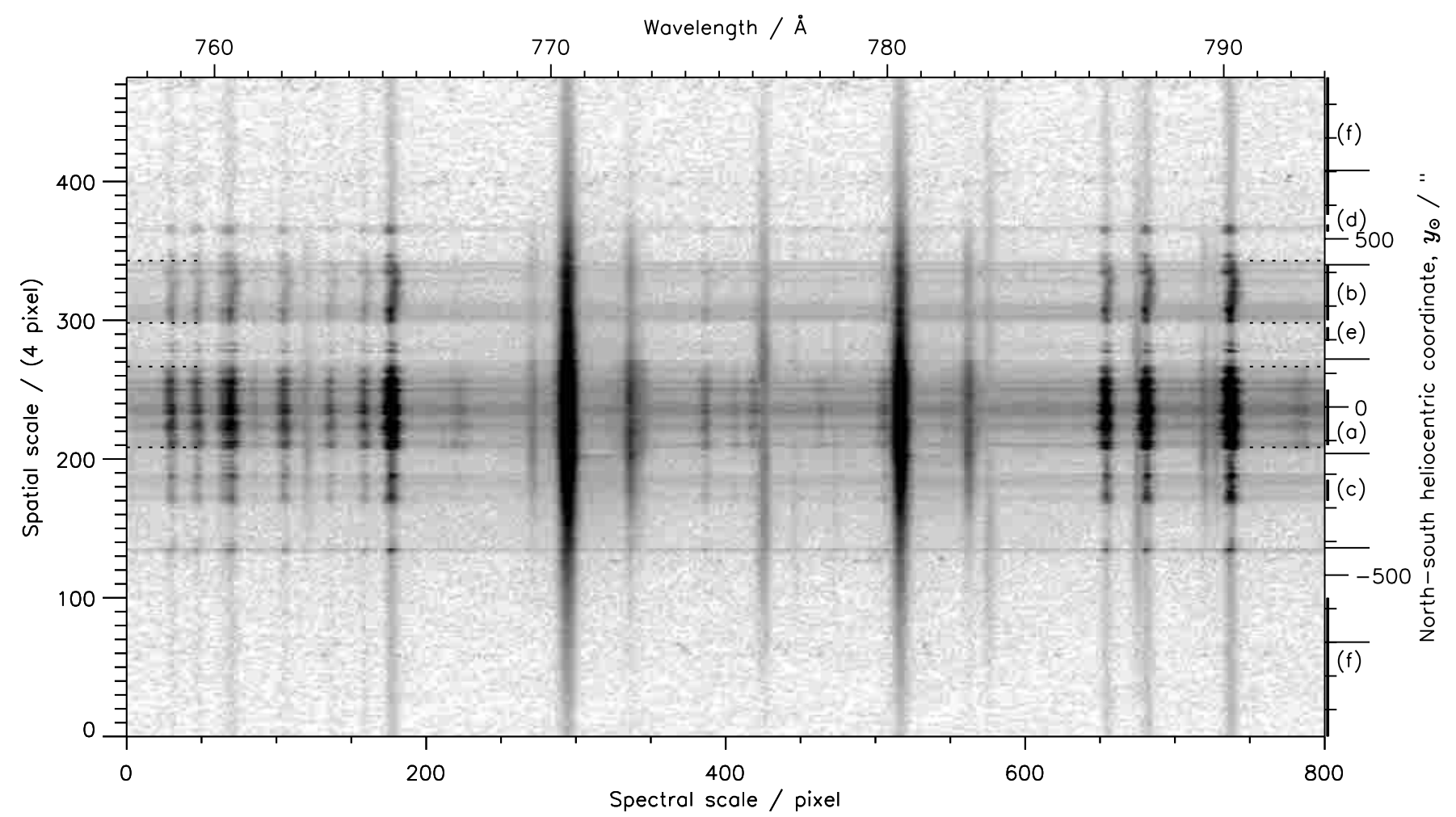

Fig. 4. North-south cross section of the east streamer. The sharp boundaries of transition region lines are marked by dotted lines at $y \odot=-120^{\prime \prime}, 120^{\prime \prime}, 250^{\prime \prime}$ and $435^{\prime \prime}$. Note that two edges (near $y \odot=-420^{\prime \prime}$ and $140^{\prime \prime}$ ) are slightly corrupted by an imperfect match of the slit sections. The coronal lines, e.g., NeVIII $(\lambda \lambda 770,780), \mathrm{Mg}$ VIII $(\lambda \lambda 772,782))$ are much smoother than the transition region lines. The coronal lines Sx $(\lambda 776)$ and S XI $(\lambda 782)$ are even stronger outside the bright central region (a). Range (b) exhibits Doppler shifts of up to $37 \mathrm{~km} \mathrm{~s}^{-1}$. The EIT image at $304 \AA$ (Fig. 5) reveals that a large prominence was present in this region. The definition of the ranges (a) to (f) for which amplitude spectra are plotted in Fig. 8 is different from that of Fig. 3, and is matched to the specific configuration of the corona. Artifacts at $y_{\odot} \approx 680^{\prime \prime},-450^{\prime \prime}$ and $-720^{\prime \prime}$ are detector noise due to the high gain setting of the amplifier.

The central regions of high intensity have, in addition to their spatial structure, very sharp edges towards larger and smaller $y_{\odot}$ coordinates. At least some of them do not coincide with slit transition (in Fig. 3 at $y_{\odot} \approx 240^{\prime \prime}$ and in Fig. 4 at $y_{\odot}=-127^{\prime \prime}$, for instance) and cannot be caused by bad fits. These sharp edges can also be seen in Fig. 6, where the radiances of Ne VIII and N IV lines are compared for both streamers. We want to point out that the different behaviour of the coronal and transition region lines at high $y_{\odot}$ can be used to estimate the influence of straylight and ascertain that the Ne VIII lines are not significantly affected by scattered light. The distinct regimes visible in Figs. 3 and 4, motivated us to present in Figs. 7 and 8 spatially averaged amplitude spectra for the ranges (a) to (f) shown in the right margins. First of all, this presentation provides a much better insight into the radiometric situation, which indicates that all lines and the H I Lyman continuum are weaker in the west. Inspection of the EIT observations on February $6 / 7$, 1998 leads to the conclusion that this difference is, in all likelihood, not related to the Sun. Instead, a slight offset (a few seconds of arc) in the $x_{\odot}$ pointing could be responsible for the asymmetric configuration (cf. Fig. 1).
Figures 7 and 8 also show the lines which are blended and consequently require special care. This holds, in particular, for Ne VIII ( $\lambda 780)$ and Mg VIII $(\lambda 772)$. An interesting question is which lines exhibit the structured behaviour and which do not. Wilhelm (1999) found that lines with formation temperatures above $\approx 4 \times 10^{5} \mathrm{~K}$ had a smooth north-south profile, whereas lines below $2.5 \times 10^{5} \mathrm{~K}(\mathrm{OV})$ displayed spatial variations. The lines O VI $(\lambda \lambda 1032,1037)$ displayed both features.

There are several density-sensitive and temperaturesensitive line pairs available in the wavelength range from $758 \AA$ to $793 \AA$ for density and temperature diagnostics (cf. Table 2). The ratio of the OV $(\lambda \lambda 759,761)$ pair depends on the electron density (Curdt et al. 1997), so does that of the Mg VIII $(\lambda \lambda 772,782)$ pair (Laming et al. 1997), while the O IV $(\lambda \lambda 779.91,787.74)$ lines form a temperature-sensitive pair. The $\mathrm{OV}$ line at $759.44 \AA$ is hopelessly blended by a S IV line at $759.34 \AA$. It is fortunate that there are other SIV lines close by in the spectrum, for instance, at $750.225 \AA$ and $753.74 \AA$. Kelly (1987) gives relative radiance ratios for the S IV lines, allowing us to estimate the contribution of the S IV line to the Ov/S IV blend to be approximately $30 \%$. 

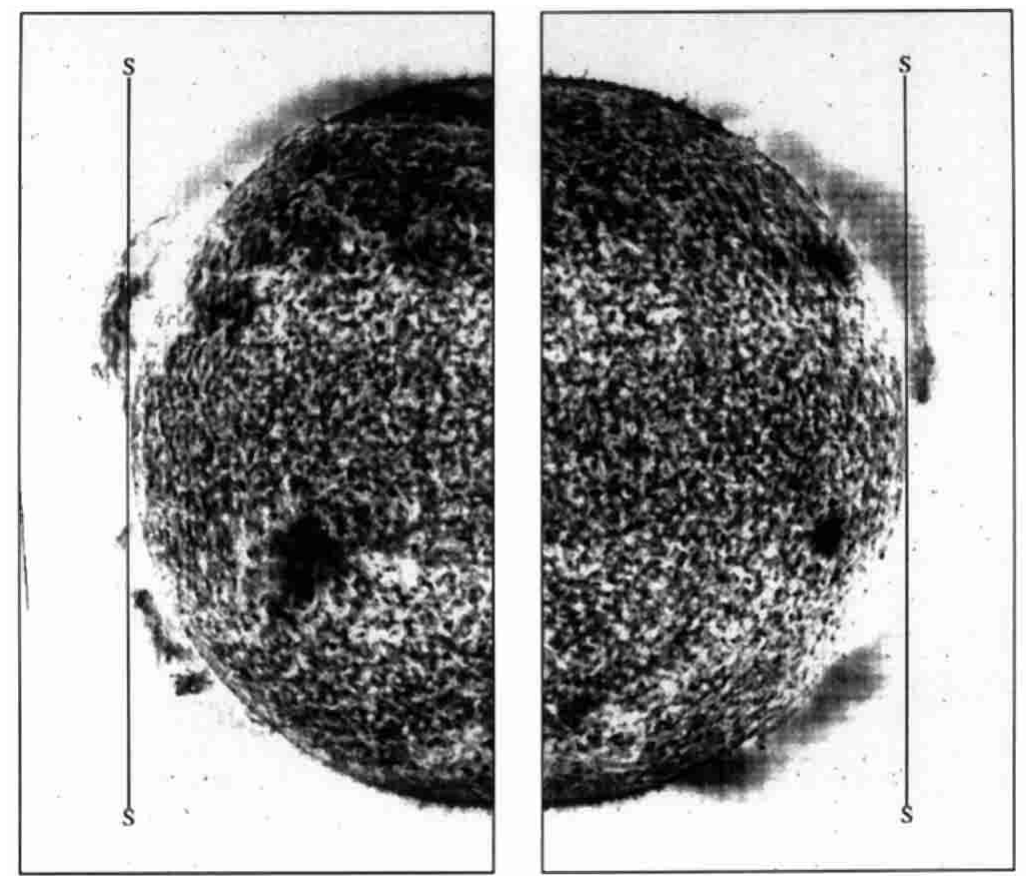

Fig. 5. The EIT spectral window around $304 \AA$ with the prominent He II line is shown for the western hemisphere at 19:19 UT on February 6 and for the eastern hemisphere at 07:19 UT on February 7, 1998. The cross sections of the streamer regions covered by the SUMER slit scans are indicated (S-S). Note the reversed grey scale, emitting areas are represented in dark.

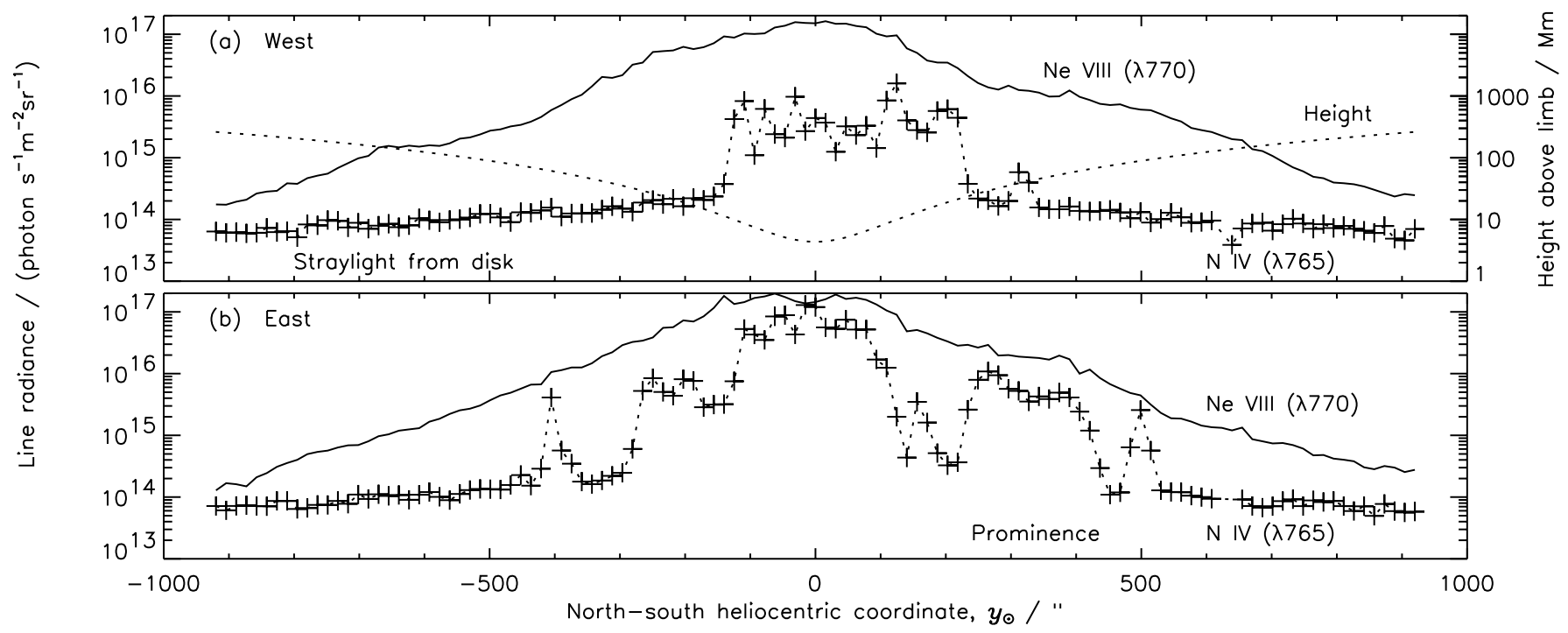

Fig. 6. Comparison of the Ne VIII ( $\lambda 770)$ line with the N IV ( $\lambda 765)$ line in both streamers a) west; b) east. The sharply bounded regions with significant contributions from the N IV transition region line are very prominent. Outside these regimes most of the radiation in N IV is caused by scattering from solar disk emission on the imperfections of the SUMER telescope mirror. The radiometric uncertainty of these measurements is $20 \%(1 \sigma)$ (Wilhelm et al. 2000b). The height of closest approach of the LOS above the limb, $h=\sqrt{x_{\odot}^{2}+y_{\odot}^{2}}-r_{0}$, is plotted as a dotted line with a scale on the right-hand side. The minimum of this height was nominally $5 \mathrm{Mm}$.

A temperature-sensitive line ratio useful in this wavelength range is $R_{1}=L(779.91) / L(787.74)$ of the O IV spectrum, where $L\left(\lambda_{0}\right)$ denotes the radiance of a line at $\lambda_{0}$. It is rather difficult to estimate the ratio $R_{1}$, because the Ne VIII (780) line is severely blending the $779 \AA$ line, which in itself is a multiplet. With constrained Gauss fits, we could separate the O IV lines from Ne VIII, but could not resolve the four members of the multiplet. We can thus only determine the ratio $R_{2}=\left(\sum L(779)\right) / L(787)$. The $779.91 \AA$ and $779.99 \AA$ lines (from the same upper level ${ }^{2} D_{5 / 2}$ ) have a branching ratio of 1:0.07, the $779.74 \AA$ and $779.82 \AA$ lines (from ${ }^{2} D_{3 / 2}$ ) have a ratio of $0.12: 1$ and the radiance ratio $(L(779.91)+$ $L(779.99)) /(L(779.74)+L(779.82))$ at $n_{\mathrm{e}}=1 \times 10^{10} \mathrm{~cm}^{-3}$ 


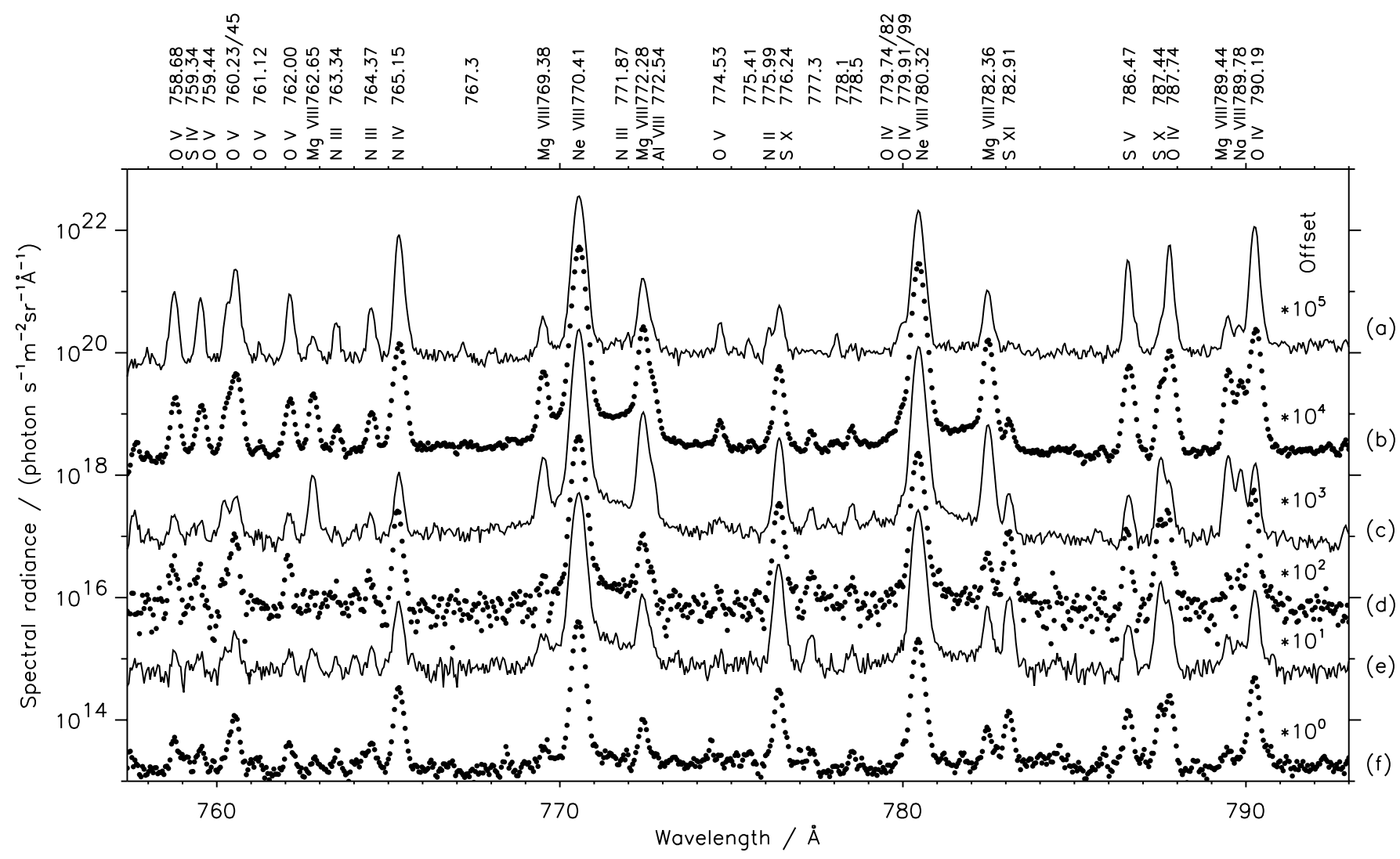

Fig. 7. Amplitude spectra of the data presented in Fig. 3 for the west streamer averaged over certain spatial regimes as indicated by the lower case letters (a) to (f). For display purposes the curves are offset by powers of 10 as shown on the right, but are radiometrically calibrated. Line identifications are provided in the upper margin. In the context of this paper note, in particular, the Ne VIII and Mg VIII lines, but also the high-temperature lines S X and S XI and their relative strengths compared to cooler lines (N II, O IV and Mg VIII). Some lines of interest are blended ( Mg VIII at $772 \AA$, Ne VIII at $780 \AA$ ) and the methods to separate them from the blends are discussed in the text. For some intense lines, notably Ne viII at $770 \AA$ in spectra (b) and (c), there is a background increase of $\approx 0.2 \%$ of the peak intensity at wavelengths a little longer than the centre wavelength. This increase is related to the high gain used for the detector and is of instrumental origin. The artifact has very little impact on the line generating it, but it must be considered, if weaker lines ride on these extensions as, for instance, $\mathrm{Mg}$ VIII $(\lambda 772)$.

and $T_{\mathrm{e}}=1.6 \times 10^{5} \mathrm{~K}$ is 1.49 (Laming 1998). Consequently we get $L(779.74): L(779.82): L(779.91): L(779.99)=$ 0.12:1.00:1.56:0.11.

The $\mathrm{Mg}$ VIII ratio is also very difficult to use, because of blends of the line at $772 \AA$ (Wilhelm \& Bodmer 1998). It was found that densities derived from $\mathrm{Mg}$ VIII were systematically higher than those from Si VIII, even after detailed considerations of possible blend contributions. The Si VIII pair of the forbidden lines at $1440 \AA$ and $1445 \AA$, on the other hand, has been widely used for coronal density diagnostics at heights of more than $15 \mathrm{Mm}$ above the limb (Doschek et al. 1997; Wilhelm et al. 1997a, 1998; Doyle et al. 1998) and yields densities of $\approx 1 \times 10^{8} \mathrm{~cm}^{-3}$ at $\approx 15 \mathrm{Mm}$ in coronal holes falling off to $1 \times 10^{7} \mathrm{~cm}^{-3}$ at about $110 \mathrm{Mm}$. The lowest value seen was $3.7 \times 10^{6} \mathrm{~cm}^{-3}$ at $210 \mathrm{Mm}$ (Doschek et al. 1997). At altitudes lower than $15 \mathrm{Mm}$ the Si VIII method does not work reliably in coronal holes, because the less intense line at $1440 \AA$ is swamped by the background radiation. The densities in streamers are higher by a factor of roughly two at comparable altitudes and thus the method can be applied down to lower heights.

The only useful line pair for coronal temperatures in the SUMER wavelength range appears to be MgIX $(\lambda \lambda 706,750)$ (Keenan et al. 1984; Wilhelm et al. 1998). This pair is however weakly density sensitive, and thus the electron density has to be known.

Consequently, we have analysed the spectral ranges containing the MgIX $(\lambda \lambda 706,750)$ lines (Bands 1 and 2 out of 12 ; cf. Table 1$)$ and the Si VIII $(\lambda \lambda 1440,1445)$ lines (Band 11) in the same manner as the range around $770 \AA$. We have then produced diagrams similar to those of Figs. 3 and 4 to check for data completeness and consistency. It turned out that the Mg IX lines at $x_{\odot}=+989^{\prime \prime}$ and $y_{\odot}=+279^{\prime \prime}$ are not available in the final telemetry data, and an interpolation was required between adjacent positions. As the diagrams do not show anything new compared to Figs. 3 and 4, they are not reproduced here. In what follows the data will be further binned into $115^{\prime \prime}$ sections in order to allow us to obtain reliable ratios at 


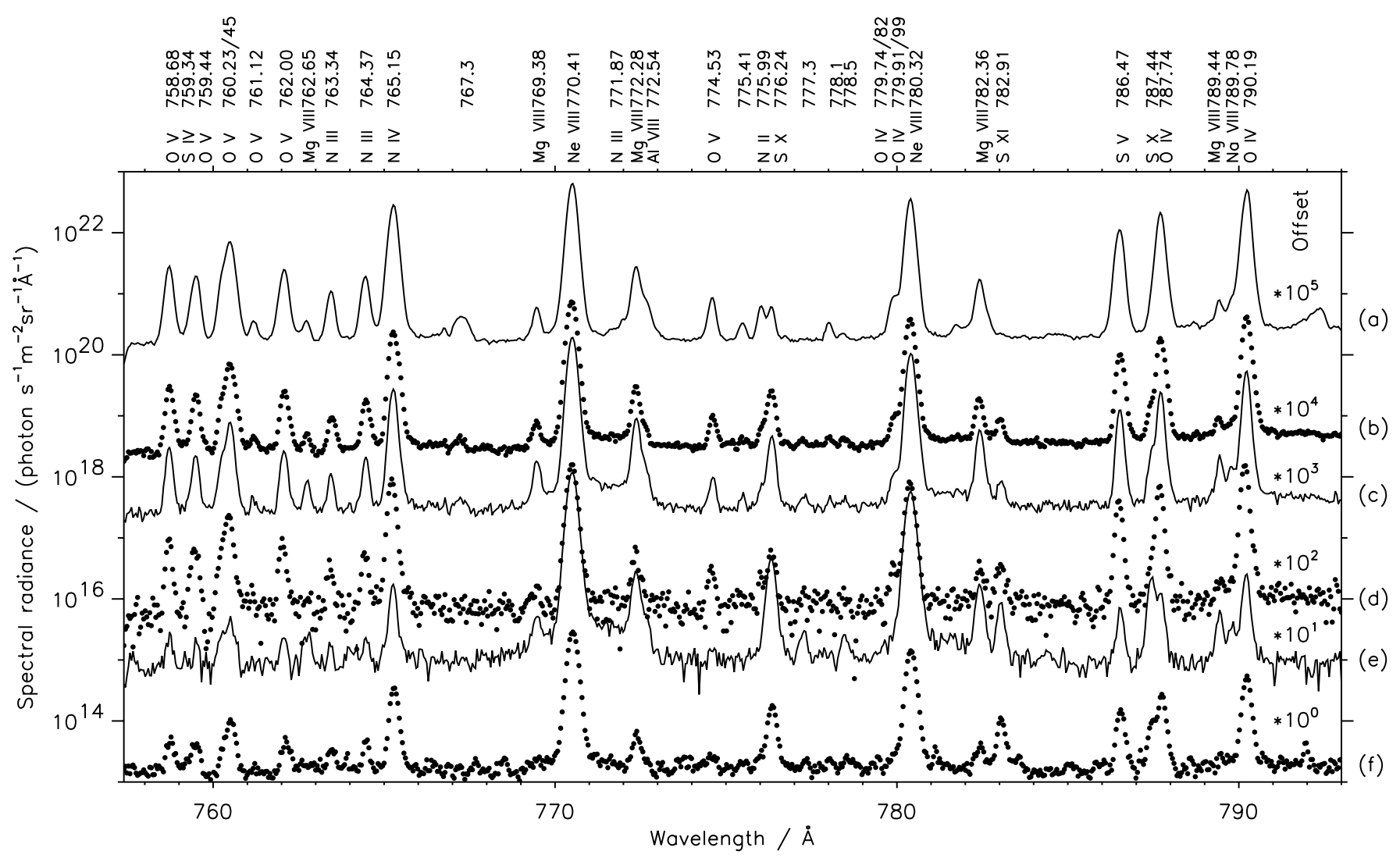

Fig. 8. Same as Fig. 7, but for the streamer in the east shown in Fig. 4. All lines, in particular the transition region ones, and the H I Lyman continuum of the spectrum (a), are stronger (by factors of two to five) than the corresponding values of the west streamer. As discussed in the text, this is probably caused by a slight difference in pointing.

large $y_{\odot}$. The complete original data sets are in the public domain and can be obtained from of the SOHO archive at http://soho.nascom.nasa.gov/data/ or from http://www.linmpi.mpg.de/english/projekte/sumer/ FILE/SumerEntryPage.html.

\subsection{The $L A S C O / C 1$ coronagraph}

LASCO is a package of three coronagraphs $\mathrm{C} 1, \mathrm{C} 2$ and C3 on SOHO. Together they image the corona from $r=1.1 R_{\odot}$ to $30 R_{\odot}$. Detailed descriptions of LASCO are given by Brueckner et al. (1995) and Socker et al. (1996). The LASCO Lyot coronagraph, C1, is designed to provide coronal images between $1.1 R_{\odot}$ and $3.2 R_{\odot}$. Key elements of the optical train of this coronagraph include a symmetric folding reflecting telescope with a lowscatter primary mirror, a tunable passband Fabry-Perot filter with an associated blocking filter set, a polaroid filter set and a photon-noise-limited CCD detector with a $1024 \times 1024$-pixel format. The optical configuration with its filter passband is such that the system operates as an imaging spectrograph. It can produce monochromatic images over the entire field of view with a spectral resolution of about $0.7 \AA$. Its spatial resolution is primarily determined by the detector pixel size with respect to the plate scale. The equivalent pixel size of the CCD is 5 ". 8 . The flux limiting aperture of the system is the Lyot stop, which results in a Rayleigh diffraction-limited resolution of $3^{\prime \prime} .3$ at $5303 \AA$.

The blocking filter selects a spectral neighbourhood of a specific emission line and suppresses all but a single transmitted interferometer order. This filter minimizes the out-of-band continuum to less than 1 part in $10^{5}$. The full width at half maximum (FWHM) of the interferometer passband has been adjusted to the expected line widths of the coronal lines. The coronagraph $\mathrm{C} 1$ was designed to study the corona in the forbidden lines Fe XIV $5303 \AA$, Caxv $5649 \AA$, and Fex $6374 \AA$, as well as in the lines Na I $5890 \AA$ and $\mathrm{H}$ I Ba $\alpha$ at $6562 \AA$. The white-light continuum can also be observed at any wavelength between $5300 \AA$ and $6400 \AA$.

Here we use only the Fex and FexIV forbidden line observations. Their thermal equilibrium formation temperatures (under equilibrium conditions) are $\approx 1 \times 10^{6} \mathrm{~K}$ and $1.8 \times 10^{6} \mathrm{~K}$, respectively. The hot Fe XIV emission is usually observed much stronger and decreases less rapidly with distance from the Sun than the Fe X emission. This behaviour can also be observed in Figs. 9 and 10 where the respective observations for February 6 and 7, 1998 are shown. Another typical observation in the Fe XIV line is the absence of emission from the coronal regions above coronal holes. At the time the present observations were made, these sectors correspond largely with the polar latitudes. We also typically see an enhanced emission from middle 

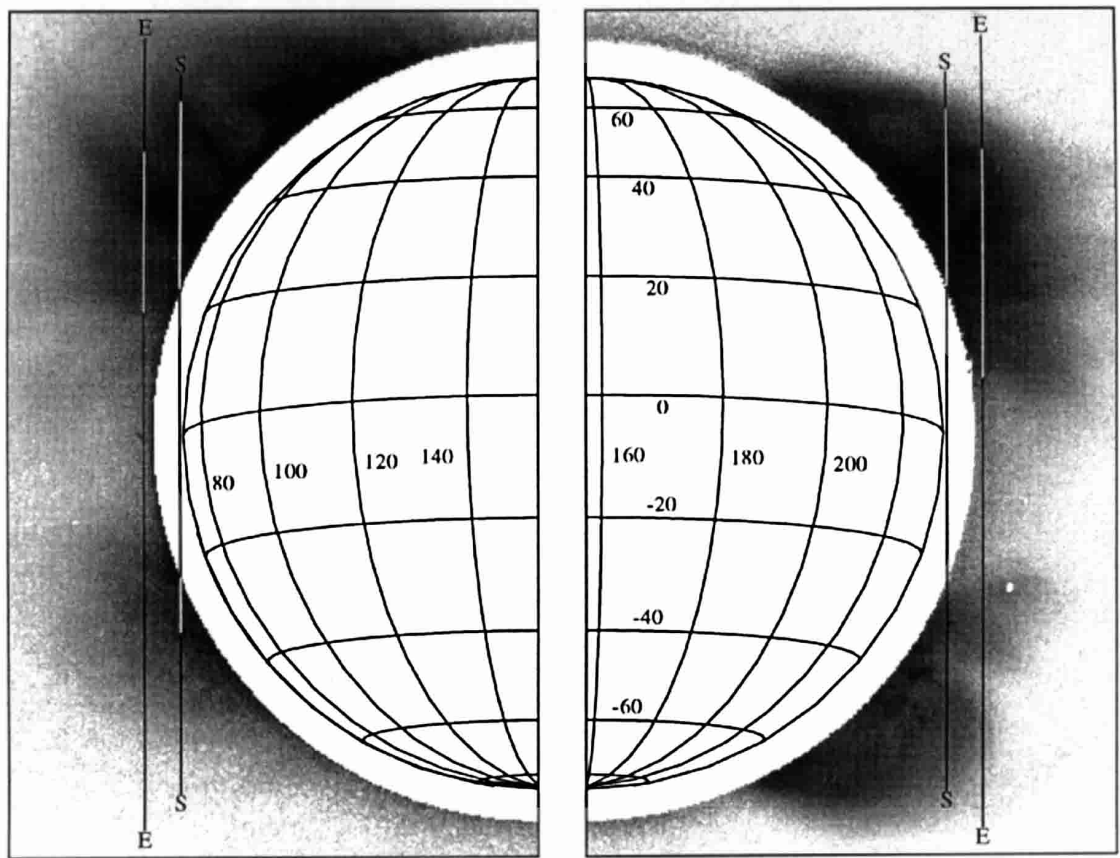

Fig. 9. LASCO/C1 Fe XIV observations $\left(r>1.1 R_{\odot}\right)$ on February 6 at 21:24 UT (western hemisphere) and on February 7,1998 at 07:32 UT (eastern hemisphere) with the north-south scans of the SUMER slit (S-S) superposed. The Sun's position and size is displayed by the Carrington longitude-latitude grid inside the occulter. Since the SUMER slit crosses the LASCO C1 occulter, we also use an extrapolated slit (E-E) parallel to the SUMER slit but at 1.1 times the distance from the centre of the solar disk. The intensity cross sections and the ratio of Fe XIV/Fex along these slits have been determined in Figs. 13 and 14 .
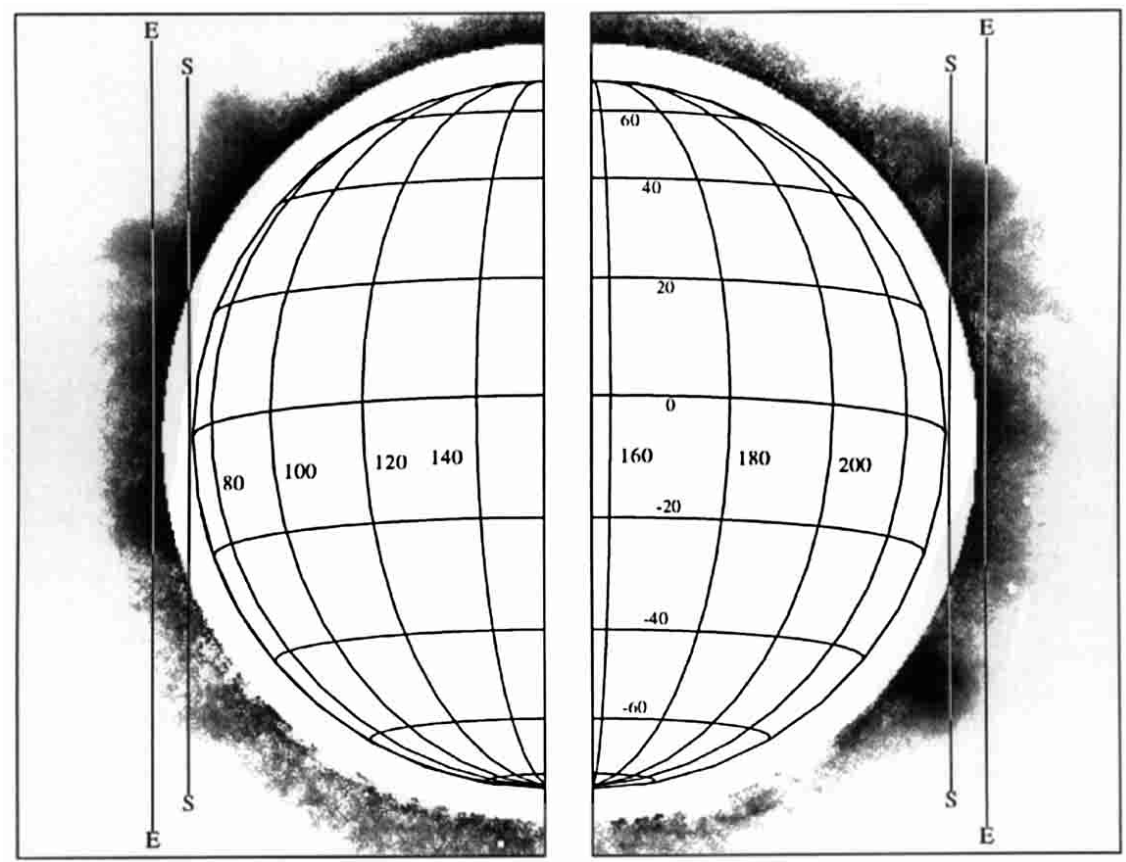

Fig. 10. LASCO/C1 Fex observations on February 6 at 22:15 UT (western hemisphere) and on February 7, 1998 at $08: 15$ UT (eastern hemisphere). The Carrington longitude-latitude grid on the Sun's surface, the SUMER slit (S-S) and the extrapolated slit (E-E) are shown as in Fig. 9.

latitudes especially from regions adjacent to the coronal hole boundary while from the low latitudes the emission is only modest unless a low-latitude active region happens to pass the limb. In this respect, the observations shown in Figs. 9 and 10 are representative for the Sun at low and medium activity. In Figs. 9 and 10, the positions of the SUMER slit (S-S) are indicated on either side. Unfortunately, these slits pass through the coronagraph occulter so that there is no LASCO data available for a direct comparison at the central sections of the SUMER 
slits. To have a rough idea of how the Fex and Fe XIV emission varies along these missing parts of the slits, we show the LASCO data also along two extrapolated slits (E-E) which are parallel to the SUMER slits but have 1.1 times their distance from the center of the solar disk.

\subsection{The EIT telescope}

The Extreme-ultraviolet Imaging Telescope (EIT) provides wide-field images of the corona and transition region with 2 ". 6 pixels and up to $1.4 R_{\odot}$ from the centre of the disk. The telescope uses two normal incidence multilayercoated mirrors to define four different bandpasses. The response is selected to peak on emission lines produced either in the transition region (He II $304 \AA$, cf. Fig. 5), cool corona (Fe IX/X $171 \AA$ and Fe XII $195 \AA$ ), or hotter corona (Fe XV $284 \AA$ ). First results from EIT, including the temperature sensitivity of the four bandpasses, are described by Moses et al. (1997). The temperature response for the dominant lines as mentioned above can be seen from the corresponding contribution functions in Fig. 2 .

We construct a model differential emission measure (DEM) map, using the EIT images from the four channels which cover the temperature range from $80000 \mathrm{~K}$ to $2.2 \times 10^{6} \mathrm{~K}$. Calculation of the differential DEM curve using the EIT has been discussed in Cook et al. (1999, 2001). Briefly, we have modified the existing CHIANTI programme of Dere et al. (1997), which computes a model spectrum using an arbitrary assumed DEM curve and a large bank of atomic data, to predict radiances observed through the four channels of EIT. We use the observed radiances to modify an initial trial DEM so that it best reproduces the observations in each pixel, giving a DEM map of the entire field of view. The DEM provides a compact summary of the solar atmospheric structural information as a function of the electron temperature. In Fig. 12 we show pixel-by-pixel ratios of DEM $\left(\log T_{\mathrm{e}} / \mathrm{K}=\right.$ 6.3) $/ \mathrm{DEM}\left(\log T_{\mathrm{e}} / \mathrm{K}=6.0\right)$ for February $6 / 7,1998$. These maps nicely display areas of high and low temperature emissions.

\section{Results}

From the SUMER streamer spectra we can extract the Si VIII $(\lambda 1440)$ line down to about $7^{\prime \prime}(5 \mathrm{Mm})$, the lowest nominal altitude reached by the slit projection. It is, however, not possible to identify and measure the lines at the extreme values of $y_{\odot}$. We have also evaluated the $\mathrm{Mg}$ VIII line ratio, but found that the measured ratio had to be reduced by $25 \%$ in order to arrive at densities consistent with the Si VIII values. Laming et al. (1997) found good agreement between densities obtained from Si VIII $(\lambda \lambda 1440,1445)$ and Mg VIII $(\lambda \lambda 772,782)$, but observed the Mg VIII lines in second order with detector $\mathrm{A}$. This provided higher spectral resolution and allowed a clear separation of the Mg VIII ( $\lambda 772.28)$ and Al VIII ( $\lambda 772.54)$ lines. This would point to an underestimation of the Al viII blend. The detector background stemming from the

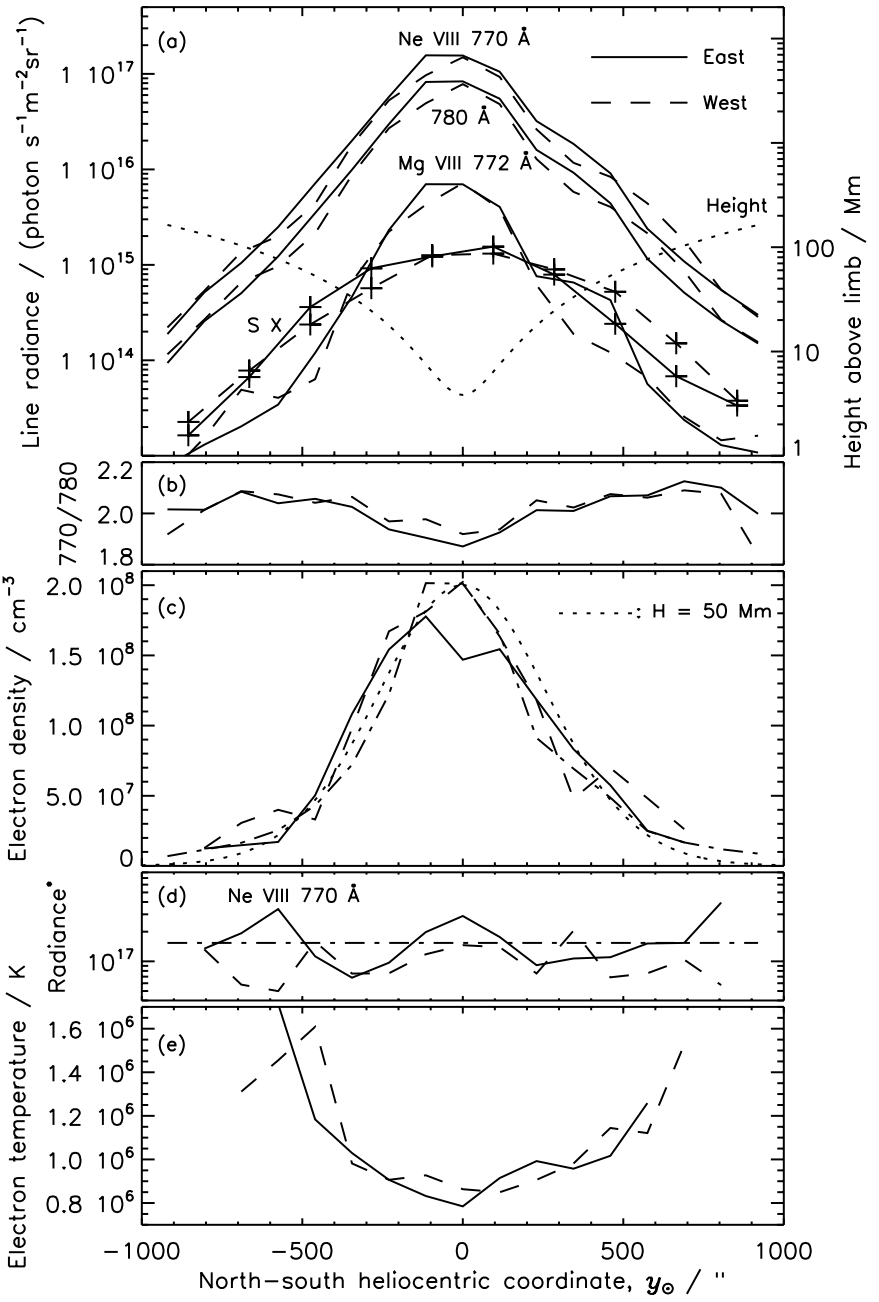

Fig. 11. a) Radiances, $L$, of the Ne VIII $(\lambda \lambda 770,780)$ lines as a function of the $y_{\odot}$ coordinate at fixed $x_{\odot}= \pm 989^{\prime \prime}$ for both the east and the west streamer. Also shown are the radiances of the Mg VIII $(\lambda 772)$ and $\mathrm{Sx}(\lambda 776)$ spectral lines. The height of closest approach, $h$, is plotted as a dotted line with a scale on the right-hand side. b) Ratios of the radiances of the Ne VIII $(\lambda \lambda 770,780)$ lines in both streamers. c) Electron densities, $n_{\mathrm{e}}$, deduced from Si viII line ratio measurements, and a calculation based on a scale height of $50000 \mathrm{~km}$ and a maximum density of $2 \times 10^{8} \mathrm{~cm}^{-3}$. Also shown as dashed-dotted curve are the densities required to obtain a constant normalized radiance in the next panel. d) Under the assumption of no changes in elemental abundance and of the ionization stages, the measured radiance values, $L$, can be reduced to a radiance, $L^{*}$, at the maximum of the density. The "density-scaled" graphs $\left(L \approx n_{\mathrm{e}}^{2}\right)$ should, with the above assumptions, be constant, which they are only in an approximate fashion as shown for the Ne VIII ( $\lambda 770)$ line. The dashed-dotted curve corresponds to that in panel c). e) Electron temperature, $T_{\mathrm{e}}$, versus the $y_{\odot}$ coordinate as obtained from the $\operatorname{Mg} \operatorname{IX}(\lambda \lambda 706,750)$ line ratio.

Ne VIII ( $\lambda 770)$ line (cf. Figs. 7 and 8 ) has been taken into account. A detailed study of the Mg VIII line ratio observations with SUMER is in preparation.

In Fig. 11, the SUMER results are presented for both streamer cross sections. The ratios of the radiances of the Ne VIII lines in panel a) are very close to a value of two at 

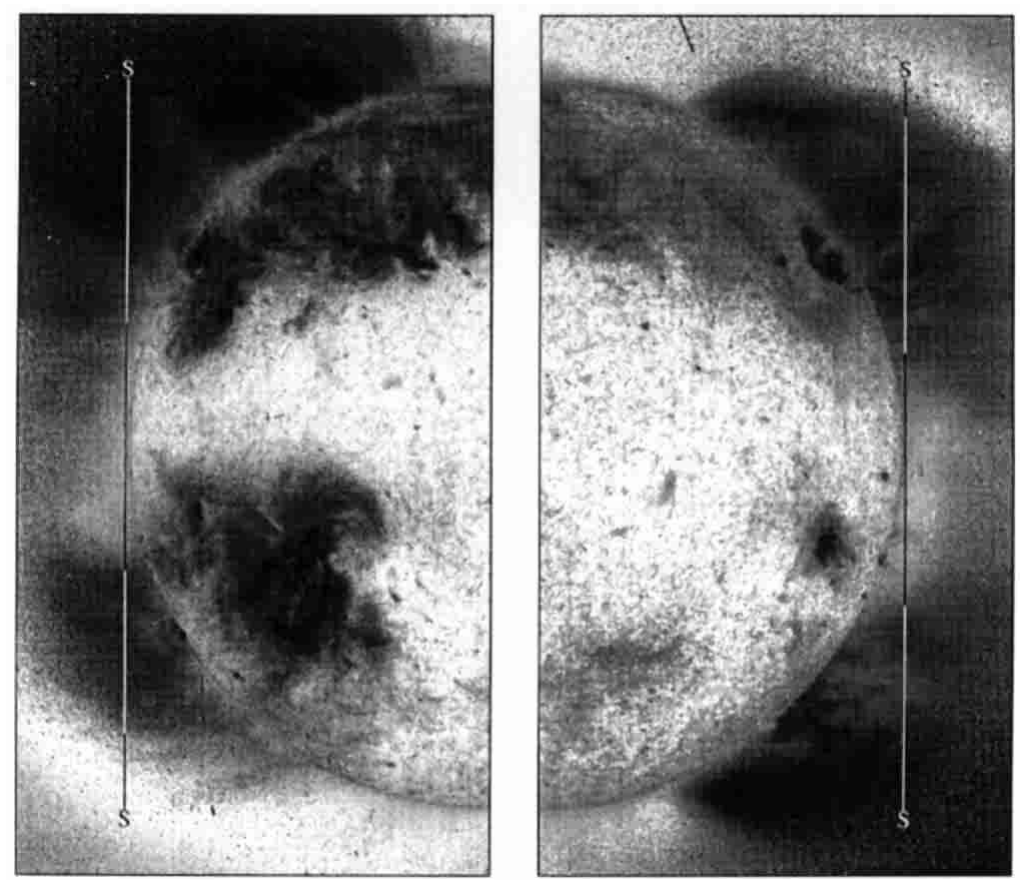

Fig. 12. Maps of $\operatorname{DEM}\left(\log T_{\mathrm{e}} / \mathrm{K}=6.3\right) / \mathrm{DEM}\left(\log T_{\mathrm{e}} / \mathrm{K}=6.0\right)$ on 6 February 1998 (western hemisphere) and on 7 February 1998 (eastern hemisphere) derived from EIT observations in all four bandpasses. Bright areas represent relatively cool plasma and dark features correspond to hot regions of the corona. The SUMER slit positions are indicated on either side.

all positions for both scans, as can be seen from panel b) It can thus be concluded that the Ne viII resonance lines are predominantly collisionally excited by electron impact and not resonantly scattered under the prevailing conditions (cf. Noci et al. 1987; Wilhelm et al. 1997b). Opacity effects observed for Ne VIII $(\lambda 770)$ in the limb-brightened regime of the solar disk by Doschek et al. (1998) are not significant in our case, although a slight systematic decrease of the ratio is discernible close to the limb. Consequently, we can assume for Ne VIII with good approximation a radiance of $L \sim n_{\mathrm{e}}^{2}$. The electron densities, $n_{\mathrm{e}}$, as a function of $y_{\odot}$ are shown in panel c) where we did not plot the last two measurements on either side, because the Si viII method does not provide accurate values at such low densities. Strictly speaking, the density is determined from the Si VIII ratio observed along the LOS, but the steep decrease of the electron density with height leads to a dominating influence of the region closest to the limb. Density-scaled radiances of the Ne VIII $(\lambda 770)$ line normalized to $2 \times 10^{8} \mathrm{~cm}^{-3}$ are given in panel $\mathrm{d})$. With the above assumptions, the radiances should be constant, which they are obviously not in a perfect sense, but variations in radiance over nearly three orders of magnitude in panel a) are reduced to about a factor of eight. Alternatively, we have constrained the normalized radiance of Ne VIII $(\lambda 770)$ to a constant value of $1.54 \times 10^{17}$ photon $\mathrm{s}^{-1} \mathrm{~m}^{-2} \mathrm{sr}^{-1}$ (the mean value of the normalized radiances) and determined the electron density required to achieve this result. This electron density is then plotted in panel c) as dashed-dotted curve. It appears to be consistent with the Si viII data.

The evaluation of the ratio of the $\mathrm{Mg}$ IX $(\lambda \lambda 706,750)$ lines was hampered by the low signal strength of the weak
$750 \AA$ line at $\left|y_{\odot}\right|$ values above $700^{\prime \prime}$. At low latitudes, an electron temperature $T_{\mathrm{e}} \approx 9 \times 10^{5} \mathrm{~K}$ was deduced. It showed a general increase towards higher latitudes as can be seen from Fig. 11e. It is important to find some verification for these Mg IX temperatures. In Fig. 11a we have added some radiance data of the $\mathrm{Mg}$ VIII $(\lambda 772)$ and $\mathrm{S} x(\lambda 776)$ lines in both streamers (cf. Table 2 for relevant formation temperatures). These measurements are obviously consistent with higher temperatures at larger $\left|y_{\odot}\right|$. The same impression can also be obtained by inspecting the $\mathrm{Mg}$ VIII and $\mathrm{Sx} / \mathrm{xI}$ lines as a function of $y_{\odot}$ in Figs. 3 and 4 . The increase of the electron temperature towards higher latitudes is thus well established, but it is not possible to decide from this data set whether the temperature depends mainly on latitude or on height. This information can, however, be obtained from EIT and LASCO observations. The EIT temperature map in Fig. 12 clearly demonstrates that there is a much greater latitudinal temperature gradient along the SUMER slit direction than perpendicular to it. At low altitudes near the equator temperatures appear to be comparable to polar coronal hole values. A quantitative estimate of the electron temperature from both line ratio and differential emission measure calculation based on EIT data leads to a values of $T_{\mathrm{e}}=1 \times 10^{6} \mathrm{~K}$ to within $10^{5} \mathrm{~K}$.

In Fig. 13 the line intensities and the ratios along the cross sections are plotted from LASCO1/C1 observations presented in Figs. 9 and 10. Given the different contribution functions of the Fex and FexIV lines this ratio should also reflect the electron temperature of the emitting plasma. The ratios on the slit profiles and on the projected profiles in Fig. 13 are only significantly different in 


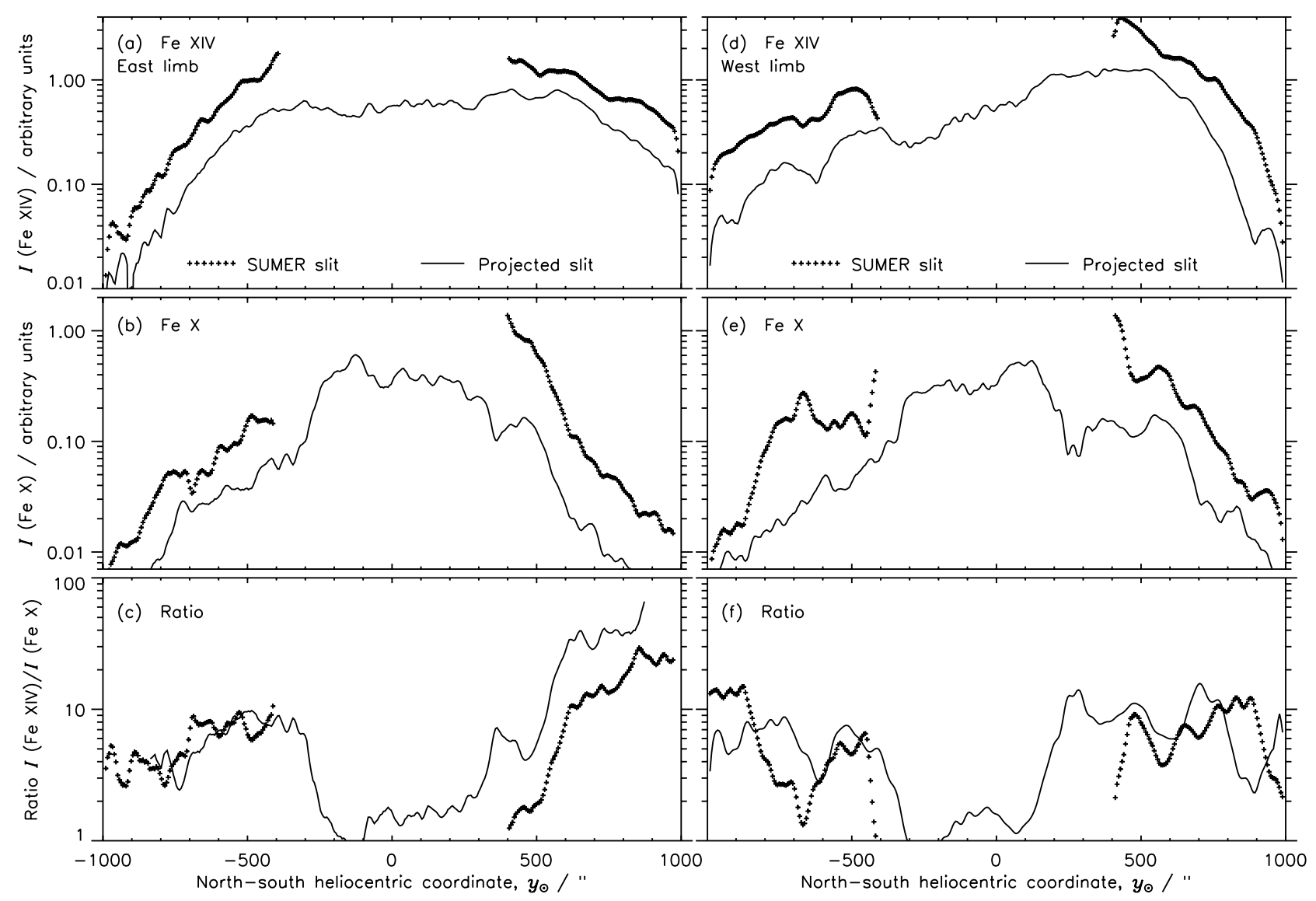

Fig. 13. Intensities of the Fe XIV line in the east streamer along the slit positions and the extrapolated projection are shown in panel a) Panel b) displays the corresponding intensities for the Fex line, whereas panel c) gives ratios of $I(\mathrm{Fe} x \mathrm{IV}) / I(\mathrm{Fe} \mathrm{x})$ for both the slit and and extrapolated positions. The panels $\mathbf{d})-\mathbf{f}$ ) display the corresponding results for the west streamer.

the northern portion of the east streamer, in all other cases the change in temperature with height between profiles $(\mathrm{S}-\mathrm{S})$ and $(\mathrm{E}-\mathrm{E})$ is small. The LASCO/C1 data, therefore, support the EIT result that there is a strong latitudinal effect.

Figure 11 can only be adequately interpreted after considering of the LOS effects involved in obtaining the line radiances. This is relatively easy for lines with formation temperatures below $2.5 \times 10^{5} \mathrm{~K}$ (the transition region lines). From Fig. 6 it can be seen that significant contributions to the N IV radiance stem from low altitudes only (if we disregard the prominence contribution in the east). Figures 3 and 4 demonstrate that all transition region lines behave in a very similar manner. Ne VIII and $\mathrm{Mg}$ VIII, on the other hand, do neither exhibit the spatial structure at low altitudes nor the cutoff towards higher latitudes. It is thus clear that these lines are emitted from a plasma volume which is much more extended, and LOS effects could be of greater importance. Can they cause the smooth latitudinal dependence of Ne VIII, for instance? This question cannot be answered without additional assumptions. First we note that the electron densities found in Fig. 11c suggest a scale height of $\approx 50 \mathrm{Mm}$ in the low corona. Although derived from observations across the streamers, it seems reasonable to expect the same scale height at positions along the LOS direction. With the earlier assumption that the radiance contributions are proportional to $n_{\mathrm{e}}^{2}$, these contributions can be calculated for all regions of the low corona and can be sorted according to their sources. It turns out that for $\left|y_{\odot}\right| \leq 150^{\prime \prime}$ approximately $70 \%$ of the Ne VIII emission is from altitudes below $20 \mathrm{Mm}$, where also most of the spatially structured transition region lines originate (cf. Fig. 6). Hence, both the structured transition region lines and most of the smooth radiation of Ne VIII (and presumably Mg VIII) are emitted from this plasma volume, which consequently has to have regions of different temperatures and, as we will see below, of different densities, too. It is also evident that the structures emitting the transition region lines must be much more limited in their spatial extent and irregular in their distribution than the hotter plasma volumes.

These findings support model calculations based on Normal Incidence X-ray Telescope (NIXT) observations, which had to assume an intermingling of chromospheric and coronal temperatures in order to produce an adequate fit between predictions and measurements (Daw et al. 1995).

The evaluations of the O IV and Ov line ratios and the resulting electron densities and temperatures are given in Table 3 for those locations along the streamers cross sections where the ratios could be determined with confidence. Comparisons with the Si VIII and MgIX results 
Table 3. Evaluation of the density-sensitive line ratio of O V $(\lambda \lambda 759.44,761.12)$ and the temperature-sensitive line ratio of O IV $(\lambda \lambda 787.73,779.91)$. The streamer locations refer to those indicated in Figs. $3,4,7$, and 8 by lower case letters in parentheses.

\begin{tabular}{|c|c|c|c|c|}
\hline \multirow{3}{*}{$\begin{array}{l}\text { Streamer } \\
\text { location }\end{array}$} & \multicolumn{2}{|c|}{$\mathrm{O} v$ ratio } & \multicolumn{2}{|c|}{ O IV ratio } \\
\hline & $L(759.44) / L(761.12)$ & Electron density $^{a}$ & $L(779.91) / L(787.73)$ & Electron temperature $^{b}$ \\
\hline & & $n_{\mathrm{e}} / \mathrm{cm}^{-3}$ & & $T_{\mathrm{e}} / \mathrm{K}$ \\
\hline \multicolumn{5}{|l|}{ West } \\
\hline (a) Brightest region & 8.8 & $2 \times 10^{9}$ & 0.039 & $3.0 \times 10^{5}$ \\
\hline (b) Structured region & 6.7 & $5 \times 10^{9}$ & $-^{c}$ & - \\
\hline \multicolumn{5}{|l|}{ East } \\
\hline (a) Structured region & 6.6 & $5 \times 10^{9}$ & 0.032 & $2.2 \times 10^{5}$ \\
\hline (b) Prominence (North) & 6.0 & $6 \times 10^{9}$ & 0.032 & $2.2 \times 10^{5}$ \\
\hline (c) Southern enhancement & 8.0 & $3 \times 10^{9}$ & 0.039 & $3.0 \times 10^{5}$ \\
\hline
\end{tabular}

${ }^{a}$ Evaluated using Fig. 7 of Curdt et al. (1997) with $T_{\mathrm{e}} \approx 2.5 \times 10^{5} \mathrm{~K}$.

${ }^{b}$ Evaluated using Fig. 9 of Curdt et al. (1997) with $n_{\mathrm{e}} \approx 1 \times 10^{10} \mathrm{~cm}^{-3}$.

${ }^{c} L(779)$ very uncertain; ratio outside useful range.

indicate that the electron density of the structured plasma regime is more than an order of magnitude higher than that of the coronal regions and the electron temperature is about a factor of three less. Hence the plasma pressure, $p_{\mathrm{e}}=n_{\mathrm{e}} T_{\mathrm{e}}$, is higher in the structured features than in the coronal regions, and the magnetic pressure has to be smaller, if the different regions are assumed to be in pressure balance.

\section{Discussion and conclusions}

Bright, apparently closed-loop systems at latitudes between $30^{\circ}$ and $45^{\circ}$ in both hemispheres were characteristic signatures of the minimum corona below $r=1.5 R_{\odot}$ in LASCO/C1 images (Schwenn et al. 1997). Using a ratio technique of the FeXII and FeIX/X channels of EIT, Moses et al. (1997) constructed temperature maps of the solar disk and the low corona, which indicated high-temperature regimes in these mid-latitude regions. Guhathakurta \& Fisher (1994) had identified these hightemperature zones using ground-based observations of the Fe XIV and Fe $\mathrm{x}$ forbidden lines. Employing essentially two different methods (studying lines with different formation temperatures and performing an evaluation of line ratios of the same ion), we also find these high-temperature regions with EIT, LASCO/C1 and SUMER at these latitudes in the inner corona in February 1998. The Mg IX line ratio method allows us to determine the electron temperature. We find approximately $T_{\mathrm{e}}=1.4 \times 10^{6} \mathrm{~K}$ at an altitude of $80 \mathrm{Mm}\left(r=1.11 R_{\odot}\right)$. There are indications that these regions have very low neon-to-magnesium elemental abundance (Wilhelm 1999).

The electron temperatures we derived close to the equatorial limb are near $9 \times 10^{5} \mathrm{~K}$ and thus are very low. They may not represent pure coronal levels as the observations of all transition region lines demonstrate a pronounced spatial structure in these regimes. The temperature thus varies considerably and our estimate may at best represent an average value for this height. The low temperatures are supported though by the EIT temperature maps of Fig. 12 which show no increase with height in this regime. The EIT data suggest an average temperature of $1 \times 10^{6} \mathrm{~K}$ with an uncertainty of $10^{5} \mathrm{~K}$. In the northern portion of the east streamer an extended prominence is present and confuses the spectra in Fig. 4. Closer to the limb, the structures with electron densities between $(2$ to 6$) \times 10^{9} \mathrm{~cm}^{-3}$ and electron temperatures below $3 \times 10^{5} \mathrm{~K}$ could be caused by spicule activity (Budnik et al. 1998; Wilhelm 2000). Spectral lines with formation temperatures above about $4 \times 10^{5} \mathrm{~K}$ do not exhibit this small-scale structure, although most of their radiation is emitted from plasma volumes at altitude levels comparable to those of the sources of the transition region lines. The identification of this spatial and temperature structure of the low corona and its sharp upper boundary is a surprising result of this study.

Warren (1999) studied SUMER spectra obtained during a SOHO roll offset on 24 February 1999. At a height of about $40 \mathrm{Mm}$ above the limb and at a position angle $\alpha=120^{\circ}$, the $\mathrm{Sx}(\lambda \lambda 1213,1196)$ line-ratio diagnostic gave an electron density of $2 \times 10^{8} \mathrm{~cm}^{-3}$. Using an emission measure analysis for silicon ions with charge stages between 7 and 10 , an electron temperature of $\approx 1.6 \times 10^{6} \mathrm{~K}$ was found. At this position angle, the slit would cross our profiles at $-570^{\prime \prime}$, where we see $T_{\mathrm{e}} \approx 1.6 \times 10^{6} \mathrm{~K}$ and densities, $n_{\mathrm{e}}$, between $(2$ and 5$) \times 10^{7} \mathrm{~cm}^{-3}$ at a height of $115 \mathrm{Mm}$ (cf. Fig. 11). Assuming again a scale height of $50 \mathrm{Mm}$, our average density is a little lower than the Sx data suggest, but are fully consistent with the results of 1999 considering the different dates of observation.

Acknowledgements. The SUMER, LASCO, and EIT projects are financially supported by DLR, NASA, NRL, CNES, CNRS, SERC, and the ESA PRODEX programme (Swiss contribution). SOHO is a mission of international cooperation between ESA and NASA. 


\section{References}

Arnaud, M., \& Raymond, J. 1992, ApJ, 398, 394

Arnaud, M., \& Rothenflug, R. 1985, A\&AS, 60, 425

Brueckner, G. E., Howard, R. A., Koomen, M. J., et al. 1995, Sol. Phys., 162, 357

Budnik, F., Schröder, K.-P., Wilhelm, K., et al. 1998, A\&A, 334, 77

Cook, J. W., Newmark, J. S., \& Moses, J. D. 1999, Proc. 8th SOHO Workshop, ESA SP-446, 241

Cook, J. W., Newmark, J. S., \& Moses, J. D. 2001, ApJ, in preparation

Cranmer, S. R., Kohl, J. L., Noci, G., et al. 1999, ApJ, 511, 481

Curdt, W., Feldman, U., Laming, J. M., et al. 1997, A\&AS, 126,281

Dammasch, I. E., Wilhelm, K., Curdt, W., et al. 1999, A\&A, 346,285

Daw, A., DeLuca, E. E., \& Golub, L. 1995, ApJ, 453, 929

Delaboudinière, J.-P., Artzner, G. E., Brunaud, J., et al. 1995, Sol. Phys., 162, 291

Denne, B., \& Hinnov, E. 1984, Phys. Rev. A, 29, 3442

Dere, K. P., Brueckner, G. E., Howard, R. A., et al. 1997, Sol. Phys., 175, 601

Dere, K. P., et al. 1997, A\&AS, 125, 149

Doschek, G. A., Warren, H. P., Laming, J. M., et al. 1997, ApJ, 482, L109

Doschek, G. A., Feldman, U., Laming, J. M., et al. 1998, ApJ, 504,573

Doyle, J. G., Mason, H. E., \& Vernazza, J. E. 1985, A\&A, 150, 69

Doyle, J. G., Banerjee, D., \& Perez, M. E. 1998, Sol. Phys., 181, 91

Edlén, B. 1983, Phys. Scr., 28, 483

Edlén, B. 1984, Phys. Scr., 30, 135

Edlén B. 1985, Phys. Scr., 31, 345

Feldman, U. 1998, Space Sci. Rev., 85, 227

Feldman, U., Behring, W. E., Curdt, W., et al. 1997, ApJS, 113,195

Feldman, U., Dammasch, I. E., \& Wilhelm, K. 2000, Space Sci. Rev., 93, 411

Geiss, J., Gloeckler, G., von Steiger, R., et al. 1995, Science, 268, 1033

Guhathakurta, M., \& Fisher, R. R. 1994, Sol. Phys., 152, 181

Hassler, D. M., Dammasch, I. E., Lemaire, P., et al. 1999, Science, 283,810

Kaufman, V., \& Martin, W. C. 1993, J. Phys. Chem. Ref. Data, 22,279

Keenan, F. P., Kingston, A. E., Dufton, P. L., et al. 1984, Sol. Phys., 94, 91
Kelly, R. L. 1987, J. Phys. Chem. Ref. Data, 16, 1

Krieger, A. S., Timothy, A. F., \& Roelof, E. C. 1973, Sol. Phys., 29,505

Laming, J. M. 1998, private communication

Laming, J. M., Feldman, U., Schühle, U., et al. 1997, ApJ, 485, 911

Lemaire, P., Wilhelm, K., Curdt, W., et al. 1997, Sol. Phys., 170,105

Mariska, J. T. 1992, The Solar Transition Region (Cambridge University Press, Cambridge)

Moses, D., Clette, F., Delaboudinière, J.-P., et al. 1997, Sol. Phys., 175, 571

Noci, G., Kohl, J. L., \& Withbroe, G. L. 1987, ApJ, 315, 706

Plunkett, S. P., Brueckner, G. E., Dere, K. P., et al. 1997, Sol. Phys., 175, 699

Raymond, J. C., Kohl, J. L., Noci, G., et al. 1997, Sol. Phys., 175,645

Ridgeley, A., \& Burton, W. M. 1972, Sol. Phys., 27, 280

Schwenn, R. 1990, in Physics of the Inner Heliosphere, vol. I, ed. R. Schwenn, \& E. Marsch (Springer-Verlag, Berlin, Heidelberg, Germany), 99

Schwenn, R., Inhester, B., Plunkett, S. P., et al. 1997, Sol. Phys., 175, 667

Simnett, G. M., Tappin, S. J., Plunkett, S. P., et al. 1997, Sol. Phys., 175, 685

Socker, D. G., Brueckner, G. E., Korendyke, C. M., et al. 1996, in ed. D. Rust, Mission to the Sun, SPIE, 2804, 126

Vourlidas, A., Subramanian, P., Dere, K. P., et al. 2000, ApJ, 534,456

Warren, H. P. 1999, Sol. Phys., 190, 363

Wilhelm, K. 1999, in Solar Wind Nine, ed. S. R. Habbal, R. Esser, J. V. Hollweg, \& P. A. Isenberg, AIP CP471, 269

Wilhelm, K. 2000, A\&A, 360, 351

Wilhelm, K., \& Bodmer, R. 1998, Space Sci. Rev., 85, 371

Wilhelm, K., Curdt, W., Marsch, E., et al. 1995, Sol. Phys., 162,189

Wilhelm, K., Lemaire, P., Curdt, W., et al. 1997a, Sol. Phys., 170, 75

Wilhelm, K., Lemaire, P., Feldman, U., et al. 1997b, Appl. Opt., 36, 6416

Wilhelm, K., Marsch, E., Dwivedi, B. N., et al. 1998, ApJ, 500, 1023

Wilhelm, K., Dammasch, I. E., Marsch, E., et al. 2000a, A\&A, 353,749

Wilhelm, K., Schühle, U., Curdt, W., et al. 2000b, Metrologia, 37,393

Woch, J., Axford, W. I., Mall, U., et al. 1997, Geophys. Res. Lett., 24, 2885

Wu, S. T., Guo, W. P., Andrews, M. D., et al. 1997, Sol. Phys., 175,719 